\title{
Modulated crystals and almost periodic measures
}

\author{
Jeong-Yup Lee ${ }^{1,2} \cdot$ Daniel Lenz ${ }^{3} \cdot$ Christoph Richard $^{4}$ (D) Bernd Sing ${ }^{5}$. \\ Nicolae Strungaru ${ }^{6,7}$
}

Received: 13 November 2019 / Revised: 23 October 2020 / Accepted: 30 October 2020 /

Published online: 17 November 2020

(c) The Author(s) 2020

\begin{abstract}
Modulated crystals and quasicrystals can simultaneously be described as modulated quasicrystals, a class of point sets introduced by de Bruijn in 1987. With appropriate modulation functions, modulated quasicrystals themselves constitute a substantial subclass of strongly almost periodic point measures. We re-analyze these structures using methods from modern mathematical diffraction theory, thereby providing a coherent view over that class. Similar to de Bruijn's analysis, we find stability with respect to almost periodic modulations.
\end{abstract}

Keywords Diffraction · Modulated crystal · Model set · Cut-and-project scheme · Almost periodic measure

Mathematics Subject Classification 52C23 $\cdot 37$ A25 $\cdot 37$ B10 $\cdot 37$ B50

\section{Introduction}

In 1964, Brouns et al. performed an X-ray diffraction experiment on a washing soda crystal, which was expected to behave like a perfect crystal. Surprisingly, a slight anomaly in its diffraction pattern was found [17]. It was later suggested to describe such anomalies by modulated crystals [21,31]. Over the years, the crystallography of such structures has been worked out and has found many applications including protein crystallography [43]. Numerous important contributions to the field have been made by Ted Janssen [64]. Recent overviews of modulated structures and of other types of aperiodic crystals are Janssen [34] and Janner and Janssen [32]. See also the monographs by Janssen et al. [33] and by van Smaalen [67] for a detailed discussion of aperiodic crystals from physical and crystallographic perspectives.

On the mathematical side, Bombieri and Taylor [15] suggested studying modulated lattices in 1985 , as they appeared to have properties similar to quasicrystals, a funda-

We dedicate this work to Michael Baake on the occasion of his 60th birthday.

Extended author information available on the last page of the article 
mentally different type of aperiodic crystal that had been discovered shortly before. Models for quasicrystals were obtained by the so-called cut-and-project construction [23,40]. De Bruijn introduced modulated quasicrystals in Euclidean space [20], a class of point sets that is nowadays subsumed by so-called deformed weighted model sets. Whereas this class comprises weighted cut-and-project sets, de Bruijn showed that it also includes certain modulated lattices [20, Sec. 5,6]. De Bruijn's Fourier analysis relied on a particular class of smooth weight functions of unbounded support, which cannot readily be extended beyond Euclidean space.

Deformed weighted model sets have further been studied by Hof [30] and by Bernuau and Duneau [13], who coined the name, see also [32]. In the meantime, it was realized that cut-and-projects sets are in fact model sets as introduced earlier by Meyer [48,50,51]. Also Euclidean space was generalized to a locally compact abelian group, and dynamical systems techniques were introduced as a powerful tool $[35,59,60]$. In that setting, deformed weighted model sets (in fact much more generally deformed weighted Delone sets) were analyzed by Baake and Lenz [8]. Modulated structures were studied by Sing $[62,63,70]$, by reconstructing the internal space of the underlying deformed model set from the diffraction pattern of the modulated structure. Let us stress that there is no universally agreed definition of modulation or deformation at present.

As mathematical diffraction theory has now reached a certain maturity, see, e.g., $[3,47,52,57]$ for recent expositions of various parts, it seems appropriate to re-analyze modulated crystals within that general framework. Whereas mean almost periodicity of the underlying point set emerges to be characteristic of pure point diffraction in general, modulated crystals are examples of more restrictive strongly almost periodic point sets. For that reason, we adopt the setting of strongly almost periodic (SAP) measures on locally compact abelian groups in our article [26,52]. On a conceptual level, diffraction has recently been studied for more general weakly almost periodic measures [47]. In particular, it is known that such measures are pure point diffractive, as their autocorrelation is strongly almost periodic [47, Thm. 7.5(a)]. Also, their diffraction amplitudes can be computed as the squared modulus of their Fourier-Bohr coefficients [47, Thm. 7.5(b)].

Although our motivation is modulations of lattices and of general ideal crystals, we will profit from the considerably more general setting of deformed weighted model sets. Hence, in this article, we discuss deformed weighted model sets as a substantial subclass of SAP measures. We will prove that deformed weighted model sets with continuous and compactly supported weight and deformation functions are strongly almost periodic, thereby extending older results for weighted model sets $[11,45,58,65,66]$. We will then deform such structures using continuous almost periodic modulations. We will prove that such modulations of deformed weighted model sets stay within the class of deformed weighted model sets, which is in line with de Bruijn's analysis [20]. We will also show that in Euclidean space, the class of deformed weighted model sets in fact coincides with the class of modulated weighted model sets. In retrospect, this justifies de Bruijn's terminology. Our diffraction analysis relies on dynamical systems techniques based on the so-called torus parametrization $[6,38,39,45,59,60]$. In particular, it yields short alternative proofs of previous results. 
Our examples also shed some light on Lagarias' question [42]: Which Delone Dirac combs are strongly almost periodic? Whereas finite local complexity enforces crystallinity [37, Cor. 5.6], deformed weighted model sets provide many examples of infinite local complexity, e.g., almost periodic modulations of lattices or of ideal crystals.

Let us briefly mention two lines of problems within that context. It would be interesting to classify SAP measures. But at present we do not even know of a simple characterization of SAP Dirac combs. Note that such combs need not be modulated crystals: think, for example, of the incommensurate structure $\mathbb{Z} \cup \alpha \mathbb{Z}$ where $\alpha$ is irrational [3, Ex. 9.6]. Whereas this point set is not uniformly discrete, it is possible to define uniformly discrete variants [3, Ex. 9.7] and [67, Ch. 4]. There are other examples on the line, arising as factors of the Kronecker flow on the two-dimensional torus [37, Sec. 6], which fail to be modulations of the integer lattice.

Another aspect concerns modulation functions beyond almost periodic ones. Consider the deterministic displacement model $\{n+\varepsilon \cdot \operatorname{frac}(\alpha n): n \in \mathbb{Z}\}$ for $\alpha \notin \mathbb{Q}$, see [3, Ex. 9.8] or [20, Sec. 6], where frac $(x)$ denotes the fractional part of $x$. This model is not strongly almost periodic, but it is a model set [3, Ex. 9.8]. It is tempting to ask whether there is some different type of almost periodicity to cover such examples. A candidate is almost automorphicity, see, e.g., [68] and [38, Thm. 1]. More generally, the above problem might be analyzed using functions with prescribed continuity properties on the Bohr compactification [54]. The latter problem may of course also be analyzed from a dynamical perspective. Consider the hull of a point set, i.e., its translation orbit closure in a Hausdorff-type metric. The hull of any SAP point set is a group, see Lemma 2. One may now ask for which deformation functions the hull is sufficiently close to a group in order to retain pure point diffractivity.

Here is the structure of the article. As a motivating example, we discuss the diffraction of sine modulated integers. The succeeding general analysis uses mathematical diffraction theory and model sets in general locally compact abelian groups. Section 3 gives basic definitions and background on dynamical systems and diffraction theory. In Sect. 4, we treat dynamical and spectral properties of general SAP measures, from which we derive their diffraction. Although these results follow from the weakly almost periodic case, we decided to give specialized proofs for a self-contained presentation. Section 5 provides necessary background about cut-and-project schemes and model sets. Section 6 is devoted to modulations and deformations of lattices, which extend our motivating example. The examples of Sect. 6 are in fact deformed weighted model sets, which are shown to be strongly almost periodic in Sect. 7. Modulations of deformed weighted model sets are analyzed in Sect. 8. It is shown that the class of deformed weighted model sets is stable under modulation, and that in Euclidean space that class coincides with the class of modulated weighted model sets. The final Sect. 9 treats modulations of ideal crystals, a class which comprises many examples from conventional crystallography. Hence, our results in that section extend the analysis from the lattice case and specialize those for deformed model sets. In Appendix, we discuss almost periodic functions taking values in a topological group. 


\section{Sine modulated integers}

\subsection{Overview}

Consider $f: \mathbb{Z} \rightarrow \mathbb{R}$ given by $f(\ell)=\varepsilon \cdot \sin (2 \pi \alpha \ell)$ with $\varepsilon$ positive and $\alpha$ irrational. The point set $\Lambda=\{\ell+f(\ell): \ell \in \mathbb{Z}\}$ is called sine modulated integers. It is a simple example of a modulated crystal [32, Sec. 3.3]. ${ }^{1}$ Since $\alpha$ is irrational, the point set $\{f(\ell): \ell \in \mathbb{Z}\}$ is dense in $[-\varepsilon, \varepsilon]$. This holds as the point set $\{\alpha \ell \bmod 1: \ell \in \mathbb{Z}\}$ is dense in $[0,1]$, a well-known fact that may be regarded as a consequence of Weyl's equidistribution theorem, see, e.g., [41, Ch. 1, Ex. 2.1]. In fact, $f$ is an almost periodic function, see Appendix for background.

For the distance $d_{\ell}$ between points arising from two consecutive integers $\ell$ and $\ell+1$, we compute

$$
\begin{aligned}
d_{\ell} & =(\ell+1+f(\ell+1))-(\ell+f(\ell))=1+\varepsilon \cdot \sin (2 \pi \alpha(\ell+1))-\varepsilon \cdot \sin (2 \pi \alpha \ell) \\
& =1+2 \varepsilon \cdot \cos (2 \pi \alpha(\ell+1 / 2)) \sin (\pi \alpha) .
\end{aligned}
$$

We thus have $\overline{\left\{d_{\ell}: \ell \in \mathbb{Z}\right\}}=[1-2 \varepsilon \cdot|\sin (\pi \alpha)|, 1+2 \varepsilon \cdot|\sin (\pi \alpha)|]$. In particular, for $\varepsilon<1 / 2$ the set $\Lambda$ is uniformly discrete, and we may label the points $\ell+f(\ell)$ in ascending order by $\ell$. If $\varepsilon \geq 1 / 2$, then $\Lambda$ fails to be uniformly discrete. In either case $\Lambda$ has infinite local complexity, i.e., $\Lambda-\Lambda$ is not uniformly discrete.

There is a topological dynamical system $\Omega$ with shift action naturally assigned to $\Lambda$, which is called its hull. It will be constructed in Sect. 2.2. The hull contains all translates of $\Lambda$ and certain additional point sets which, loosely spoken, do not differ much from the translates. The relation between the dynamical spectrum of $\Omega$ and the diffraction spectrum of $\Lambda$ allows us to compute its diffraction explicitly in Sect. 2.3. In fact, any point set of the hull has the same diffraction. This dynamical approach is complementary to the usual one of computing the Fourier-Bohr coefficients by a limiting procedure. It is reviewed in Sect. 3.3 in a general setting.

There is a simple parametrization of the hull of sine modulated integers by a twodimensional torus $\mathbb{T}$ with induced shift action. We call the corresponding factor map $\mu: \mathbb{T} \rightarrow \Omega$ the torus parametrization ${ }^{2}$ of $\Omega$. Since the induced shift action on $\mathbb{T}$ is a dense rotation on the compact group $\mathbb{T}$, one can conclude that sine modulated integers are pure point diffractive [8, Sec. 3]. If $\varepsilon<1 / 2$, then the factor map is a homeomorphism, which implies that sine modulations are strongly almost periodic by Lemma 2. In Sect. 6, we will prove by a different method that general sine modulated integers are strongly almost periodic and hence pure point diffractive, if one properly takes into account multiplicities, compare Remark 2.

Before we do the calculations, let us describe the diffraction of sine modulated integers for $\varepsilon<1 / 2$. The Bragg peak positions lie dense in Fourier space and are

\footnotetext{
1 If $\alpha$ is rational, then the modulation function $f$ is periodic, and $\Lambda$ is a so-called ideal crystal. Such structures do not differ much from a lattice and will be discussed in Sect. 9, see especially Remark 10.

2 In the context of model sets, the name was coined in [6] for a suitable inverse of $\mu$, see also [59] and [60]. For a historical discussion and a recent dynamical approach see [38,39].
} 


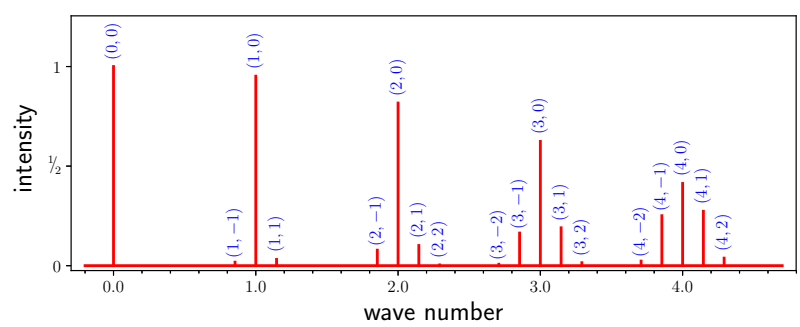

Fig. 1 Diffraction of sine modulated integers $\left(\varepsilon=1 / 20, \alpha=1 / \tau^{4}\right)$. Label $(m, n)$ corresponds to Bragg peak position $m+\alpha n$

explicitly given by $\mathbb{Z}[\alpha]=\{m+\alpha n: m, n \in \mathbb{Z}\}$. They have positive intensities

$$
\left|a_{m, n}\right|^{2}=\left|\int_{0}^{1} \mathrm{e}^{2 \pi \mathrm{i}(n s+(m+\alpha n) \varepsilon \cdot \sin (2 \pi s))} \mathrm{d} s\right|^{2} .
$$

The diffraction of sine modulated integers is depicted in Fig. 1 for $\varepsilon=1 / 20$ and $\alpha=1 / \tau^{4}$, where $\tau$ denotes the golden mean.

In general, for small modulation strength $\varepsilon>0$ one observes Bragg peaks of high intensity at the lattice positions, accompanied by Bragg peaks of small intensity. If a Bragg peak of small intensity is close to some lattice Bragg peak, it is sometimes called a satellite. In particular, this is the case if the modulation length is of a different order of magnitude than the lattice constant.

\subsection{The hull and its torus parametrization}

Recall that the point set $\Lambda \subset \mathbb{R}$ is uniformly discrete if and only if $\varepsilon<1 / 2$. In general, $\Lambda$ is locally finite, i.e., discrete and closed. In fact, $\Lambda$ is translation bounded, i.e., there exists finite constants $C$ and $R$ such that any intersection with a ball of radius $R$ contains at most $C$ points.

The hull of $\Lambda$ is the translation orbit closure of $\Lambda$ in a suitable topology, which we now describe. Let us consider the collection $\mathcal{D}=\mathcal{D}_{C, R}$ of point sets that are translation bounded with constants $C, R$. We equip $\mathcal{D}$ with the local topology, a Hausdorff type uniform topology that is frequently used when studying point set dynamical systems [7, Sec. 4]. It is generated by the entourages

$$
U_{R, \delta}:=\left\{\left(\Lambda, \Lambda^{\prime}\right) \in \mathcal{D} \times \mathcal{D}: B_{R} \cap \Lambda \subset B_{\delta}+\Lambda^{\prime} \text { and } B_{R} \cap \Lambda^{\prime} \subset B_{\delta}+\Lambda\right\},
$$

where $R, \delta$ vary over the positive real numbers. Here, $B_{r}$ denotes the centered closed ball of radius $r$. In particular, for every $\Lambda \in \mathcal{D}$, the collection of all $U_{R, \delta}(\Lambda)=\left\{\Lambda^{\prime} \in\right.$ $\left.\mathcal{D}:\left(\Lambda, \Lambda^{\prime}\right) \in U_{R, \delta}\right\}$, where $R, \delta$ vary over the positive reals, is a neighborhood system of $\Lambda$. It turns out that $\mathcal{D}$ is a compact metrizable Hausdorff space [7, Thm. 3]. In particular, we can use sequences for convergence arguments. Two point sets are close in the local topology, if they coincide on large centered balls up to small local shifts. 
Consider the hull $\Omega=\overline{\{x+\Lambda: x \in \mathbb{R}\}} \subset \mathcal{D}$ of $\Lambda$ in the local topology, with point set translation as natural $\mathbb{R}$-action. Since $\alpha$ is irrational, it is not hard to see that $\Omega=\left\{\Lambda_{r, s}:(r, s) \in \mathbb{R}^{2}\right\}$, where $\Lambda_{r, s}=\{r+\ell+\varphi(\alpha \ell+s): \ell \in \mathbb{Z}\}$ and $\varphi(x)=\varepsilon \cdot \sin (2 \pi x)$. Due to the symmetries $\Lambda_{r, s+1}=\Lambda_{r+1, s+\alpha}=\Lambda_{r, s}$, the hull is parametrized by a two-dimensional torus. We will describe in Sect. 4 how such a torus arises for strongly almost periodic structures in a natural way.

There is another natural construction of the torus, which will be central in Sect. 6: Consider the function space $H=\overline{\{f(\cdot+\ell): \ell \in \mathbb{Z}\}}$, where the closure is taken using the topology of uniform convergence. It carries a natural group structure $\dot{+}$ defined via $f(\cdot+\ell) \dot{+} f\left(\cdot+\ell^{\prime}\right)=f\left(\cdot+\left(\ell+\ell^{\prime}\right)\right)$. The LCA group $H$ is a compactification of $\mathbb{Z}$, since $f$ is almost periodic. In fact, $H=\{\varphi(s+\alpha \cdot): s \in[0,1)\}$. Thus, $H$ is homeomorphic to a one-dimensional torus. Now $\mathcal{L}=\{(\ell, f(\cdot+\ell)): \ell \in \mathbb{Z}\} \subset \mathbb{R} \times H$ is a group and a Delone set, i.e., $\mathcal{L}$ is lattice in $\mathbb{R} \times H$. Consider $\mathbb{T}=(\mathbb{R} \times H) / \mathcal{L}$ with natural $\mathbb{R}$-action $s+[r, g]=[s+r, g]$ and define $\mu: \mathbb{T} \rightarrow \Omega$ by

$$
\mu([r, g])=r+\{\ell+g(\ell): \ell \in \mathbb{Z}\} .
$$

Lemma 1 The map $\mu: \mathbb{T} \rightarrow \Omega$ is a factormap, i.e., it is continuous onto and commutes with the natural $\mathbb{R}$-actions on $\mathbb{T}$ and on $\Omega$. If $\varepsilon<1 / 2$, then $\mu$ is a homeomorphism.

Proof We show that $\mu$ is well-defined, onto and continuous. We then show that $\mu$ is an $\mathbb{R}$-map. We finally show that $\mu$ is one-to-one if $\varepsilon<1 / 2$, which implies that $\mu$ is a homeomorphism in that case since $\Omega$ is compact.

$\mu$ is well-defined: Take arbitrary $(r, g) \in \mathbb{R} \times H$ and write $g=\lim _{k \rightarrow \infty} f\left(\cdot-\ell_{k}\right)$ for some integer sequence $\left(\ell_{k}\right)_{k}$. Then $\mu([r, g]) \in \Omega$ since

$\mu([r, g])=\left\{r+\ell+\lim _{k \rightarrow \infty} f\left(\ell-\ell_{k}\right): \ell \in \mathbb{Z}\right\}=\lim _{k \rightarrow \infty}\left(r+\ell_{k}+\{\ell+f(\ell): \ell \in \mathbb{Z}\}\right)$.

The latter limit indeed exists in $\Omega$, as any accumulation point of $\left(r+\ell_{k}+\Lambda\right)_{k}$ in the compact space $\Omega$ coincides with $\mu([r, g])$. The definition is independent of the choice of representative: Let $(\ell, f(\cdot+\ell)) \in \mathcal{L}$ and note

$$
\begin{aligned}
\mu([r+\ell, g(\cdot) \dot{+} f(\cdot+\ell)]) & =r+\ell+\left\{\ell^{\prime}+g\left(\ell^{\prime}\right) \dot{+} f\left(\ell^{\prime}+\ell\right): \ell^{\prime} \in \mathbb{Z}\right\} \\
& =r+\ell+\left\{\ell^{\prime}+\lim _{k \rightarrow \infty} f\left(\ell^{\prime}-\ell_{k}\right) \dot{+} f\left(\ell^{\prime}+\ell\right): \ell^{\prime} \in \mathbb{Z}\right\} \\
& =r+\ell+\left\{\ell^{\prime}+\lim _{k \rightarrow \infty} f\left(\ell^{\prime}-\ell_{k}+\ell\right): \ell^{\prime} \in \mathbb{Z}\right\} \\
& =r+\ell+\left\{\ell^{\prime}-\ell+\lim _{k \rightarrow \infty} f\left(\ell^{\prime}-\ell_{k}\right): \ell^{\prime} \in \mathbb{Z}\right\} \\
& =r+\left\{\ell^{\prime}+g\left(\ell^{\prime}\right): \ell^{\prime} \in \mathbb{Z}\right\}=\mu([r, g]) .
\end{aligned}
$$

$\mu$ is onto: Consider any $\Lambda^{\prime} \in \Omega$. For every $k \in \mathbb{N}$ choose $r_{k} \in \mathbb{R}$ such that

$$
r_{k}+\{\ell+f(\ell): \ell \in \mathbb{Z}\} \in U_{k, \frac{1}{k}}\left(\Lambda^{\prime}\right)
$$


By passing to a suitable subsequence of $\left(r_{k}\right)_{k}$, we can find integers $\left(\ell_{k}\right)_{k}$ such that $\lim _{k \rightarrow \infty}\left(r_{k}-\ell_{k}\right)=r$ for some $r \in[0,1)$. Note that

$$
r_{k}+\{\ell+f(\ell): \ell \in \mathbb{Z}\}=r_{k}-\ell_{k}+\left\{\ell^{\prime}+f\left(\ell^{\prime}-\ell_{k}\right): \ell^{\prime} \in \mathbb{Z}\right\} .
$$

Using compactness of $H$, we may assume that $f\left(\cdot-\ell_{k}\right)$ converges, by passing to a suitable subsequence. Now define $g=\lim _{k \rightarrow \infty} f\left(\cdot-\ell_{k}\right)$. We then have $\Lambda^{\prime}=$ $r+\{\ell+g(\ell): \ell \in \mathbb{Z}\}$. Thus, $[r, g] \in \mathbb{T}$ satisfies $\mu([r, g])=\Lambda^{\prime}$.

$\mu$ is continuous: Consider an arbitrary member $\mu([r, g])$ in the hull. Fix arbitrary $R>0$ and $\varepsilon>0$ and consider $r^{\prime} \in B_{\varepsilon / 2}(r) \subset \mathbb{R}$ and $g^{\prime} \in B_{\varepsilon / 2}(g) \subset H$. By the triangle inequality, we then have

$$
\mu\left(\left[r^{\prime}, g^{\prime}\right]\right) \subset B_{\varepsilon}+\mu([r, g]), \quad \mu([r, g]) \subset B_{\varepsilon}+\mu\left(\left[r^{\prime}, g^{\prime}\right]\right) .
$$

In particular, we have $\mu\left(\left[r^{\prime}, g^{\prime}\right]\right) \in U_{R, \varepsilon}(\mu([r, g)])$. As $R>0$ and $\varepsilon>0$ were arbitrary, this shows that $\mu$ is continuous.

$\mu$ is an $\mathbb{R}$-map: Take any $s \in \mathbb{R}$ and note

$$
\mu(s+[r, g])=\mu([s+r, g])=s+r+\{\ell+g(\ell): \ell \in \mathbb{Z}\}=s+\mu([r, g]) .
$$

$\mu$ is one-to-one for $\varepsilon<1 / 2$ : Consider $\mu([r, g])=\mu([s, h])$ and assume $r \in[0,1)$ and $s=0$ without loss of generality. Write $\mu([r, g])=\left\{p_{\ell}: \ell \in \mathbb{Z}\right\}$ and $\mu([0, h])=$ $\left\{q_{\ell}: \ell \in \mathbb{Z}\right\}$, where $p_{\ell}=r+\ell+g(\ell)$ and $q_{\ell}=\ell+h(\ell)$. Note that $p_{\ell_{1}}<p_{\ell_{2}}$ and $q_{\ell_{1}}<q_{\ell_{2}}$ whenever $\ell_{1}<\ell_{2}$ since $\overline{g(\mathbb{Z})}=\overline{h(\mathbb{Z})} \subset(-1 / 2,1 / 2)$ due to irrationality of $\alpha$, and that we have

$$
p_{\ell} \in(\ell-1 / 2, \ell+3 / 2), \quad q_{\ell} \in(\ell-1 / 2, \ell+1 / 2)
$$

for every $\ell \in \mathbb{Z}$. In particular either $p_{0}=q_{0}$ or $p_{0}=q_{1}$. Assuming $p_{0}=q_{1}$, we infer $p_{\ell}=q_{\ell+1}$ for all $\ell \in \mathbb{Z}$. Thus, $r+\overline{g(\mathbb{Z})}=1+\overline{h(\mathbb{Z})}$, which is impossible as $r \in[0,1)$. We thus have $p_{0}=q_{0}$, which leads to $p_{\ell}=q_{\ell}$ for all $\ell \in \mathbb{Z}$. Thus, $r+\overline{g(\mathbb{Z})}=\overline{h(\mathbb{Z})}$, which implies $r=0$ and $g=h$. Hence, $\mu$ is one-to-one.

\subsection{Diffraction of sine modulated integers}

We compute the diffraction measure of sine modulated integers via the dynamical systems approach. The torus parametrization $\mu: \mathbb{T} \rightarrow \Omega$ can be used to embed $L^{2}(\Omega)$ isometrically into $L^{2}(\mathbb{T})$, compare [8, Thm. 1]. (For $\varepsilon<1 / 2$, the torus parametrization is a homeomorphism, and both spaces can be identified.) In particular, since $(\mathbb{T}, \mathbb{R})$ has pure point dynamical spectrum, $(\Omega, \mathbb{R})$ has pure point dynamical spectrum too, compare $[8$, Prop. 1]. The latter property is equivalent to $(\Omega, \mathbb{R})$ having pure point diffraction [7, Thm. 7].

As $(\mathbb{T}, \mathbb{R})$ is a dense rotation on a compact group, the space $L^{2}(\mathbb{T})$ admits an orthonormal basis of eigenfunctions $e_{\xi}$ with eigenvalue $\xi \in \widehat{\mathbb{R}} \cong \mathbb{R}$, compare [69]. Every such $\xi$ is a potential Bragg peak position in the diffraction of $\Lambda$. For explicit 
expressions, let us denote by $\Gamma$ the invariance lattice of $\Omega=\left\{\Lambda_{r, s}:(r, s) \in \mathbb{R}^{2}\right\}$ and its dual lattice by $\Gamma_{0}$. We have

$$
\Gamma=\left\{\left(\begin{array}{c}
m \\
n+\alpha m
\end{array}\right): m, n \in \mathbb{Z}\right\}, \quad \Gamma_{0}=\left\{\left(\begin{array}{c}
-m-\alpha n \\
n
\end{array}\right): m, n \in \mathbb{Z}\right\} .
$$

Eigenfunctions of the translation operator on $L^{2}(\mathbb{T})$, where $\mathbb{T}=\mathbb{R}^{2} / \Gamma$, arise from lattice invariant plane waves $x \mapsto \chi_{k}(x)=\mathrm{e}^{2 \pi \mathrm{i} k \cdot x}$, which restricts $k$ to elements in $\Gamma_{0}$. Given $k=(-m-\alpha n, n)^{T}$, we will write $e_{\xi}$ instead of $\chi_{k}$ for $\xi=m+\alpha n$, which will cause no confusion as $k$ can be reconstructed from $\xi$. A simple calculation shows that $e_{\xi} \in L^{2}(\mathbb{T})$ is indeed an eigenfunction of the translation operator with eigenvalue $\xi$, and $\left\{e_{\xi}: \xi \in \mathbb{Z}[\alpha]\right\}$ is total and orthonormal in $L^{2}(\mathbb{T})$. Using the isometric embedding, we can apply the diffraction formula Eq. (5) from Sect. 3.3. The diffraction intensity at $\xi$ is given by $\left|a_{\xi}\right|^{2}$, where

$$
a_{\xi}=\int_{\mathbb{T}} \overline{e_{\xi}(t)} f_{\xi \cdot \psi}(\mu(t)) \mathrm{d} t
$$

Here $\psi \in C_{c}(\mathbb{R})$ is any function satisfying $\int_{\mathbb{R}} \psi(x) \mathrm{d} x=1$, and we have used $f_{\psi}(\Lambda)=\sum_{p \in \Lambda} \psi(p)$. If $\mu$ is a homeomorphism, then $a_{\xi} \neq 0$ for all $\xi \in \mathbb{Z}[\alpha]$.

Let us now assume $\varepsilon<1 / 2$ for simplicity. In order to compute $a_{\xi}$ for $\xi=m+\alpha n$, we write

$$
\begin{aligned}
a_{\xi} & =\int_{0}^{1} \int_{0}^{1} \overline{e_{\xi}((r, s))} f_{\xi \cdot \psi}\left(\Lambda_{r, s}\right) \mathrm{d}(r, s) \\
& =\sum_{\ell \in \mathbb{Z}} \int_{0}^{1} \int_{0}^{1} \xi(-r) \mathrm{e}^{2 \pi \mathrm{i} n s}(\xi \cdot \psi)(r+\ell+\varphi(\alpha \ell+s)) \mathrm{d}(r, s) .
\end{aligned}
$$

As $\psi$ has compact support, only finitely many terms are nonzero in the above sum. Suppose now that $\psi$ is sharply concentrated about 0 . Then, up to an arbitrarily small error, the above expression simplifies to

$$
a_{\xi}=\sum_{\ell \in\{-1,0\}} \int_{0}^{1} \int_{0}^{1} \xi(\ell+\varphi(\alpha \ell+s)) \mathrm{e}^{2 \pi \mathrm{i} n s} \psi(r+\ell+\varphi(\alpha \ell+s)) \mathrm{d}(r, s) .
$$

Consider the term corresponding to $\ell=0$ in the above sum. We can have $r+\varphi(s)=0$ for some $r \in(0,1)$ only if $s \in(1 / 2,1)$. Thus, up to an arbitrarily small error, that term equals

$$
\begin{aligned}
& \int_{1 / 2}^{1} \int_{0}^{1} \xi(\varphi(s)) \mathrm{e}^{2 \pi \mathrm{i} n s} \psi(r+\varphi(s)) \mathrm{d}(r, s)=\int_{1 / 2}^{1} \xi(\varphi(s)) \mathrm{e}^{2 \pi \mathrm{i} n s} \mathrm{~d} s \\
& \quad=\int_{1 / 2}^{1} \mathrm{e}^{2 \pi \mathrm{i}(n s+(m+\alpha n) \varphi(s))} \mathrm{d} s .
\end{aligned}
$$


Next, consider the term corresponding to $\ell=-1$ in the above sum. We can have $r-1+\varphi(-\alpha+s)=0$ for some $r \in(0,1)$ only if $s \in(\alpha, \alpha+1 / 2)$. As we might choose a fundamental domain of $\Gamma$ arbitrarily for integration, that term equals

$$
\begin{aligned}
& \int_{\alpha}^{\alpha+1 / 2} \int_{0}^{1} \xi(-1+\varphi(-\alpha+s)) \mathrm{e}^{2 \pi \mathrm{i} n s} \psi(r-1+\varphi(-\alpha+s)) \mathrm{d}(r, s) \\
& \quad=\int_{\alpha}^{\alpha+1 / 2} \xi(-1+\varphi(-\alpha+s)) \mathrm{e}^{2 \pi \mathrm{i} n s} \mathrm{~d} s=\int_{0}^{1 / 2} \xi(-1+\varphi(s)) \mathrm{e}^{2 \pi \mathrm{i} n(s+\alpha)} \mathrm{d} s \\
& \quad=\int_{0}^{1 / 2} \mathrm{e}^{2 \pi \mathrm{i}(n s+(m+\alpha n) \varphi(s))} \mathrm{d} s .
\end{aligned}
$$

Combining the latter two results, we get $\left|a_{\xi}\right|^{2}=\left|a_{m, n}\right|^{2}$, compare Eq. (1).

\section{Setting and notation}

Throughout $G, H$ will denote locally compact abelian groups (LCAG) [22,24,55]. The group operation on a LCAG will be written additively as + or $\dot{+}$ if necessary to avoid misunderstandings. Given an LCA group $G$, we choose a Haar measure on $G$ and denote it by $m_{G}$ or by $\mathrm{d} t$. The dual group of $G$ is denoted by $\widehat{G}$, and the pairing between a character $\chi \in \widehat{G}$ and $t \in G$ is written as $\chi(t)$. As usual the Fourier transform $\widehat{f}$ of an integrable function $f$ is defined by $\widehat{f}(\chi)=\int_{G} \overline{\chi(t)} f(t) \mathrm{d} t$. We always choose the Haar measure on $\widehat{G}$ such that the Plancherel theorem [22, Thm. 3.4.8] holds.

\subsection{Complex Radon measures}

Whenever $X$ is a topological space the space of continuous functions on $X$ is denoted by $C(X)$, the subspace of continuous functions with compact support by $C_{c}(X)$, the space of continuous bounded functions by $C_{b}(X)$, and the subspace of uniformly continuous and bounded functions by $C_{\mathrm{u}}(X)$. The latter two spaces are complete normed spaces when equipped with the supremum norm $\|\cdot\|_{\infty}$. We will often deal with locally compact $\sigma$-compact spaces.

A topological space $X$ carries the Borel $\sigma$-algebra generated by all closed subsets of $X$. By the Riesz-Markov representation theorem, the set $\mathcal{M}(X)$ of all complex Radon measures on a locally compact space $X$ can then be identified with the dual space $C_{c}(X)^{*}$ of complex valued, linear functionals on $C_{c}(X)$ which are continuous with respect to a suitable topology, see [53, Ch. 6.5] for details. For this reason, we usually write $\int_{X} \varphi \mathrm{d} \mu=\mu(\varphi)$ for $\varphi \in C_{c}(X)$. The space $\mathcal{M}(X)$ then carries the vague topology, i.e., the weakest topology that makes all functionals $\mu \mapsto \mu(\varphi)$, $\varphi \in C_{c}(X)$, continuous. If $X$ is metrizable, then $\mathcal{M}(X)$ is metrizable as well. The convolution $\mu * v$ of two finite measures $\mu$ and $v$ on $G$ is defined to be the measure $(\mu * v)(\varphi):=\iint \varphi(s+t) \mathrm{d} \mu(s) \mathrm{d} \nu(t)$. 
By [53, Thm. 6.5.6], for each measure $\mu \in \mathcal{M}(X)$ there exists a positive measure $|\mu|$, called the total variation of $\mu$ such that for all $\varphi \in C_{c}(X)$ with $\varphi \geq 0$ we have

$$
|\mu|(\varphi)=\sup \left\{|\mu(\psi)|: \psi \in C_{c}(X),|\psi| \leq \varphi\right\}
$$

Now let $G$ be a LCAG. A measure $\mu \in \mathcal{M}(G)$ is called translation bounded if there exist some $C>0$ and an open nonempty relatively compact set $V$ in $G$ so that

$$
|\mu|(t+V) \leq C
$$

for every $t \in G$. The set of all translation bounded measures satisfying (2) is denoted by $\mathcal{M}_{C, V}(G)$. It carries the vague topology inherited from $\mathcal{M}(G)$ and is compact in that topology. The set of all translation bounded measures is denoted by $\mathcal{M}^{\infty}(G)$.

\subsection{Measure dynamical systems}

Whenever the LCAG $G$ acts on the compact Hausdorff space $\Omega$ by a continuous action

$$
\alpha: G \times \Omega \longrightarrow \Omega, \quad(t, \omega) \mapsto \alpha_{t}(\omega),
$$

where $G \times \Omega$ carries the product topology, the pair $(\Omega, \alpha)$ is called a topological dynamical system over $G$. An $\alpha$-invariant probability measure on $\Omega$ is then called ergodic if every measurable invariant subset of $\Omega$ has either measure zero or measure one. The dynamical system $(\Omega, \alpha)$ is called uniquely ergodic if there exists a unique $\alpha$ invariant probability measure on $\Omega$, which then is ergodic by standard theory. $(\Omega, \alpha)$ is called minimal if, for all $\omega \in \Omega$, the $G$-orbit $\left\{\alpha_{t} \omega: t \in G\right\}$ is dense in $\Omega$.

Given an $\alpha$-invariant probability measure $m$ on $\Omega$, we can form the Hilbert space $L^{2}(\Omega, m)$ of square integrable measurable functions on $\Omega$. This space is equipped with the inner product

$$
\langle f, g\rangle=\langle f, g\rangle_{\Omega}:=\int_{\Omega} \overline{f(\omega)} g(\omega) \mathrm{d} m(\omega)
$$

The action $\alpha$ gives rise to a unitary representation $T:=T^{\Omega}:=T^{(\Omega, \alpha, m)}$ of $G$ on $L^{2}(\Omega, m)$ by

$$
T_{t}: L^{2}(\Omega, m) \longrightarrow L^{2}(\Omega, m), \quad\left(T_{t} f\right)(\omega):=f\left(\alpha_{-t} \omega\right)
$$

for every $f \in L^{2}(\Omega, m)$ and arbitrary $t \in G$. An $f \in L^{2}(\Omega, m)$ is called an eigenfunction of $T$ with eigenvalue $\xi \in \widehat{G}$ if for every $t \in G$ we have $T_{t} f=\xi(t) f$. An eigenfunction (to $\chi$, say) is called continuous if it has a continuous representative $f$ with $f\left(\alpha_{-t} \omega\right)=\xi(t) f(\omega)$, for all $\omega \in \Omega$ and $t \in G$. The representation $T$ is said to have pure point spectrum if the set of eigenfunctions is total in $L^{2}(\Omega, m)$. One then also says that the dynamical system $(\Omega, \alpha)$ has pure point dynamical spectrum.

Let two topological dynamical systems $(\Omega, \alpha)$ and $(\Theta, \beta)$ under the action of $G$ be given. Then, $(\Theta, \beta)$ is called a (topological) factor of $(\Omega, \alpha)$, with factor map $\Phi$, 
if $\Phi: \Omega \longrightarrow \Theta$ is a continuous surjection with $\Phi\left(\alpha_{t}(\omega)\right)=\beta_{t}(\Phi(\omega))$ for all $\omega \in \Omega$ and $t \in G$.

We will be concerned with dynamical systems built from measures. These systems will be discussed next. They have been introduced in $[7,8]$, to which we refer for further details and proofs of the subsequent discussion. There is an obvious action of $G$ on $\mathcal{M}^{\infty}(G)$, again denoted by $\alpha$, given by

$\alpha: G \times \mathcal{M}^{\infty}(G) \longrightarrow \mathcal{M}^{\infty}(G), \quad(t, v) \mapsto \alpha_{t} v$ with $\left(\alpha_{t} v\right)(\varphi):=v\left(\delta_{-t} * \varphi\right)$

for $\varphi \in C_{c}(G)$. Here, $\delta_{t}$ denotes the unit point mass at $t \in G$ and the convolution $\omega * \varphi$ between $\varphi \in C_{c}(G)$ and $\omega \in \mathcal{M}^{\infty}(G)$ is defined by

$$
(\omega * \varphi)(s):=\int \varphi(s-u) \mathrm{d} \omega(u) .
$$

It is not hard to see that $\alpha$ is continuous when restricted to a compact subset of $\mathcal{M}^{\infty}(G)$.

Definition $1(\Omega, \alpha)$ is called a dynamical system on the translation bounded measures on $G$ (TMDS) if $\Omega$ is a compact $\alpha$-invariant subset of $\mathcal{M}_{C, V}(G)$ for some open relatively compact $V$ and $C>0$.

Every translation bounded measure $v$ gives rise to a TMDS $(\Omega(v), \alpha)$ [7], where

$$
\Omega(v):=\overline{\left\{\alpha_{t} v: t \in G\right\}}
$$

More precisely, if $v \in \mathcal{M}_{C, V}(G)$, then $\Omega(v) \subset \mathcal{M}_{C, V}(G)$. We call $\Omega(v)$ the hull of $v$.

\subsection{Mathematical diffraction theory}

We review elements of mathematical diffraction theory following [7,8,44,46]. Fix an LCA group $G$ and assume that $G$ is $\sigma$-compact. The, $G$ admits a van Hove sequence [60], i.e., a sequence $\left(B_{n}\right)_{n \in \mathbb{N}}$ of compact sets in $G$ such that for every compact $K \subset G$ we have

$$
\lim _{n \rightarrow \infty} \frac{m_{G}\left(\partial^{K} B_{n}\right)}{m_{G}\left(B_{n}\right)}=0 .
$$

Here for arbitrary compact $A, K \subset G$ we use the notion of van Hove boundary

$$
\partial^{K} A:=\left((K+A) \backslash A^{\circ}\right) \cup((-K+\overline{G \backslash A}) \cap A),
$$

where the bar denotes the closure of a set and the circle denotes the interior. In $G=\mathbb{R}^{d}$, the closed $n$-balls constitute a van Hove sequence. Fix $\omega \in \mathcal{M}^{\infty}(G)$, such as the Dirac comb of a point set in $G$, and consider for arbitrary $\xi \in \widehat{G}$ the average

$$
a_{\xi}=\lim _{n \rightarrow \infty} \frac{1}{m_{G}\left(B_{n}\right)} \int_{B_{n}} \overline{\xi(t)} \mathrm{d} \omega(t) .
$$


In this article, the above limit will always exist and will always be independent of the choice of the van Hove sequence. In crystallography, the number $a_{\xi}$ is called the scattering amplitude or the Fourier-Bohr coefficient. In a kinematic diffraction experiment [19], the intensity of diffraction at $\xi$ is given by $\left|a_{\xi}\right|^{2}$.

Hof [29] suggested the following mathematical framework for diffraction. Given $\omega \in \mathcal{M}^{\infty}(G)$, one first computes the autocorrelation measure

$$
\gamma_{\omega}=\lim _{n \rightarrow \infty} \frac{1}{m_{G}\left(B_{n}\right)} \omega_{B_{n}} * \widetilde{\omega_{B_{n}}}
$$

with respect to the given van Hove sequence $\left(B_{n}\right)_{n \in \mathbb{N}}$. Here the limit is taken in the vague topology, $\omega_{B_{n}}$ denotes the restriction of $\omega$ to $B_{n}$, and the reflected measure $\tilde{\mu}$ is, for $\mu \in \mathcal{M}^{\infty}(G)$, defined by $\tilde{\mu}(\varphi):=\overline{\mu\left(\overline{\varphi^{\dagger}}\right)}$, where $\varphi^{\dagger}(x)=\varphi(-x)$. In this article, the above limit will always exist and will always be independent of the choice of the van Hove sequence. More generally, this is the case if the hull $(\Omega(\omega), \alpha)$ is uniquely ergodic. ${ }^{3}$ As $\gamma_{\omega}$ is a positive definite measure by construction, its Fourier transform $\widehat{\gamma_{\omega}}$ exists as a measure. It is called the diffraction measure of $\omega$ (see [1,12,24,26,52] for definition and background on Fourier transforms of complex Radon measures). Its point part is explicitly given by

$$
\widehat{\gamma_{\omega}}(\{\xi\})=\left|a_{\xi}\right|^{2}=\left|\lim _{n \rightarrow \infty} \frac{1}{m_{G}\left(B_{n}\right)} \int_{B_{n}} \overline{\xi(t)} \mathrm{d} \omega(t)\right|^{2},
$$

see, e.g., [29, Thm. 3.4]. We say that $\omega \in \mathcal{M}^{\infty}(G)$ is pure point diffractive (relative to the given van Hove sequence) if the autocorrelation $\gamma_{\omega}$ exists and its Fourier transform $\widehat{\gamma_{\omega}}$ is a pure point measure.

We now turn to a dynamical description of diffraction $[7,44]$. Let $G$ be an arbitrary LCAG. Let $(\Omega, \alpha)$ be a TMDS, equipped with an $\alpha$-invariant measure $m$. Let $\psi \in$ $C_{c}(G)$ with $\int \psi(t) \mathrm{d} t=1$ be given. Then, $\gamma_{m}: C_{c}(G) \longrightarrow \mathbb{C}$ defined by

$$
\gamma_{m}(\varphi):=\int_{\Omega} \int_{G} \int_{G} \varphi(s+t) \psi(t) \mathrm{d} \omega(s) \mathrm{d} \tilde{\omega}(t) \mathrm{d} m(\omega),
$$

is a positive definite measure which does not depend on the choice of $\psi$. The measure $\gamma_{m}$ is called the autocorrelation measure of $(\Omega, \alpha, m)$. If $e_{\xi} \in L^{2}(\Omega, m)$ is a normalized eigenfunction of $T$ with eigenvalue $\xi \in \widehat{G}$, we then have

$$
\widehat{\gamma_{m}}(\{\xi\})=\left|\int_{\Omega} \overline{e_{\xi}(\omega)} \cdot \omega(\xi \cdot \psi) \mathrm{d} m(\omega)\right|^{2},
$$

where $\psi \in C_{c}(G)$ such that $\int_{G} \psi(t) \mathrm{d} t=1$ is arbitrary. The measure $\widehat{\gamma_{m}}$ is called the diffraction measure of $(\Omega, \alpha, m)$.

Assume now that $G$ is $\sigma$-compact and fix $v \in \mathcal{M}^{\infty}(G)$. If $(\Omega(v), \alpha)$ is uniquely ergodic, we have $\widehat{\gamma_{m}}=\widehat{\gamma_{\omega}}$ for every $\omega \in \Omega(\nu)$, compare [44, Thm. 3, Thm. 5].

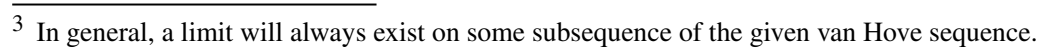


${ }^{4}$ In particular this means that the diffraction amplitudes can be computed in two different ways using Eq. (4) or Eq. (5). In this article, we will always deal with uniquely ergodic $(\Omega(v), \alpha)$. We will always compute the diffraction measure using the dynamical approach. In particular, we do not need to impose $\sigma$-compactness of $G$.

\section{Strongly almost periodic measures}

In Sect. 2, we discussed sine modulated integers. We showed that its hull is a compact abelian group, an insight which enabled us to infer the diffraction of sine modulated integers. The present section is devoted to a discussion of the general phenomenon, where we use measures instead of point sets. Note that diffraction has recently been studied for more general weakly almost periodic measures [47]. For the ease of the reader, we include proofs adapted to our simpler setting.

Consider $v \in \mathcal{M}^{\infty}(G)$, such as the Dirac comb of a Delone set in $G$. The following lemma characterizes the situation that its hull $\Omega(v)$ is a group compactification of $G$ in terms of strong almost periodicity of $v$. This is certainly well known, see, e.g., [47, Prop. 3.6] and compare [37, Thm. 3.3] and [37, Thm. 4.2] for Delone sets. We include a proof based on [45] for the convenience of the reader.

Recall that a translation bounded measure $v \in \mathcal{M}^{\infty}(G)$ is strongly almost periodic if the function $v * \varphi \in C_{\mathrm{u}}(G)$ is almost periodic for every $\varphi \in C_{c}(G)$. As usual, $f \in C_{\mathrm{u}}(G)$ is almost periodic if $\left\{\delta_{t} * f: t \in G\right\}$ is relatively compact in $C_{\mathrm{u}}(G)$. See Proposition 9 in "Appendix A" or [18,35] for background.

Lemma 2 Let $v \in \mathcal{M}^{\infty}(G)$ be given. Then, the following assertions are equivalent:

(i) The hull $\Omega(v)$ of $v$ is a compact abelian group, with the addition $\dot{+}$ satisfying $\alpha_{s} v \dot{+} \alpha_{t} v=\alpha_{s+t} v$ for any $s, t \in G$.

(ii) The measure $v$ is strongly almost periodic.

(iii) Every $\omega \in \Omega(v)$ is strongly almost periodic.

In this case, the map $\mathrm{J}: G \rightarrow \Omega(v)$ defined by $\mathrm{J}(t)=\alpha_{t} \nu$ is a continuous group homomorphism with dense range, and

$$
\alpha_{t} \omega=\jmath(t) \dot{+} \omega
$$

holds for any $t \in G$ and any $\omega \in \Omega(v)$.

Proof The equivalence between (i) and (ii) can be inferred from [45, Lemma 4.2]. For the equivalence between (ii) and (iii), consider any $\varphi \in C_{c}(G)$ and any $\varepsilon>0$. Then, all functions $\omega * \varphi \in C_{\mathrm{u}}(G)$, where $\omega$ ranges over $\Omega(v)$, have the same $\varepsilon$-almost periods.

Finally, the map is continuous with dense range. It is a group homomorphism when $\Omega(v)$ carries the group structure defined in (i). It remains to compute the action $\alpha$ in terms of $j$ : If $\omega=\alpha_{s} v$, then for any $\varphi \in C_{c}(G)$,

$$
\left(\alpha_{t} \omega\right)(\varphi)=\omega\left(\delta_{-t} * \varphi\right)=\left(\alpha_{s} v\right)\left(\delta_{-t} * \varphi\right)=v\left(\delta_{-s} * \delta_{-t} * \varphi\right)
$$

\footnotetext{
4 If $(\Omega(v), \alpha)$ fails to be uniquely ergodic but $m$ is ergodic, then the latter equality only holds almost surely with respect to $m$, by the Birkhoff ergodic theorem.
} 


$$
\begin{aligned}
& =v\left(\delta_{-s-t} * \varphi\right)=\left(\alpha_{s+t} \nu\right)(\varphi)=\left(\alpha_{t} v \dot{+} \alpha_{s} v\right)(\varphi) \\
& =(J(t) \dot{+} \omega)(\varphi) .
\end{aligned}
$$

Let $\omega=\lim _{\iota} \alpha_{s_{\iota}} v$, i.e., $\left(\alpha_{s_{\iota}} \nu\right)_{\iota}$ is a net converging to $\omega$. Then,

$$
\begin{aligned}
\left(\alpha_{t} \omega\right)(\varphi) & =\omega\left(\delta_{-t} * \varphi\right)=\lim _{\iota}\left(\alpha_{s_{\iota}} \nu\right)\left(\delta_{-t} * \varphi\right)=\lim _{\iota} v\left(\delta_{-s_{\iota}} * \delta_{-t} * \varphi\right) \\
& =\lim _{\iota}\left(\alpha_{t+s_{\iota}} \nu\right)(\varphi)=\lim _{\iota}\left(\alpha_{t} \nu \dot{+} \alpha_{s_{\iota}} \nu\right)(\varphi)=\left(\alpha_{t} \nu \dot{+} \lim _{\iota} \alpha_{s_{\iota}} \nu\right)(\varphi) \\
& =(J(t) \dot{+} \omega)(\varphi) .
\end{aligned}
$$

We can now readily derive basic spectral properties of dynamical systems associated with strongly almost periodic measures. For notation and basic results of mathematical diffraction theory, recall Sect. 3 .

Theorem 1 Let $v \in \mathcal{M}^{\infty}(G)$ be strongly almost periodic and $\mathrm{J}: G \longrightarrow \Omega(v)$ the canonical mapping described in Lemma 2. Then, the following hold.

(i) $(\Omega(v), \alpha)$ is uniquely ergodic and minimal.

(ii) For the shift operator $T$ on $L^{2}(\Omega(v))$, any character $\lambda: \Omega(v) \rightarrow S^{1}$ is an eigenfunction of $T$ to the eigenvalue $\lambda \circ \mathrm{J}: G \rightarrow S^{1}$. The set $\widehat{\Omega(v)}$ of continuous characters provides an orthonormal basis of $L^{2}(\Omega(v))$. In particular, every character is continuous, and the measure dynamical system $(\Omega(\nu), \alpha)$ has pure point dynamical spectrum.

(iii) The measure $v$ has a unique autocorrelation $\gamma$. Its Fourier transform, the diffraction measure $\widehat{\gamma} \in \mathcal{M}^{\infty}(\widehat{G})$, is pure point and given by

$$
\widehat{\gamma}=\sum_{\lambda \in \widehat{\Omega(v)}}\left|a_{\lambda \circ J}\right|^{2} \delta_{\lambda \circ J}
$$

where the constants $a_{\lambda \circ \mathrm{J}}$ are given by

$$
a_{\lambda \circ J}=\int_{\Omega(v)} \overline{\lambda(\omega)} \omega(\lambda \circ J \cdot \varphi) \mathrm{d} \omega
$$

and $\varphi \in C_{c}(G)$ is any function satisfying $\int_{G} \varphi(t) \mathrm{d} t=1$.

Remark 1 If $v$ is a Dirac comb, it is possible to interpret minimality geometrically in terms of the underlying point set. The underlying point set is almost repetitive [25, Thm. 3.11].

Proof (i) Let $m$ be any $G$-invariant probability measure on the hull $\Omega(v)$. Let $f \in$ $C(\Omega(v))$ be arbitrary. Then, we have for every $t \in G$ the equalities

$$
\int f(J(t) \dot{+} \omega) \mathrm{d} m(\omega)=\int f\left(\alpha_{-t} \omega\right) \mathrm{d} m(\omega)=\int f(\omega) \mathrm{d} m(\omega),
$$


as $m$ is $G$-invariant. Since $J(G)$ is dense in $\Omega(v)$ and $f$ is continuous on $\Omega(v)$, we have

$$
\int f(\sigma \dot{+} \omega) \mathrm{d} m(\omega)=\int f(\omega) \mathrm{d} m(\omega)
$$

for every $\sigma \in \Omega(v)$. Thus, $m$ is a Haar measure. Since $m$ is a probability measure, $m$ is unique. This implies that $\Omega(v)$ is minimal, see, e.g., [69, Thm. 6.17]. Here is a direct argument: Assume $\omega=\lim _{\iota} \alpha_{t_{l}} v \in \Omega(v)$. Then, $\Omega(\omega) \subset \Omega(v)$ by translation invariance and closedness of $\Omega(v)$. It is also readily seen that $v=\lim _{\iota} \alpha_{-t_{\iota}} \omega$, which implies $\Omega(v) \subset \Omega(\omega)$. Thus, $\Omega(\omega)=\Omega(v)$ for all $\omega \in \Omega(v)$, which shows minimality.

(ii) Let $\lambda: \Omega(v) \rightarrow S^{1}$ be any character. Then,

$$
\left(T_{t} \lambda\right)(\omega)=\lambda\left(\alpha_{-t} \omega\right)=\lambda(J(t) \dot{+} \omega)=\lambda(J(t)) \lambda(\omega)
$$

for every $\omega \in \Omega(v)$. Thus, $\lambda \in L^{2}(\Omega(v))$ is an eigenfunction of $T$ to the eigenvalue $\lambda \circ$ $J$. By the Peter-Weyl theorem [61], the continuous characters provide an orthonormal basis of $L_{2}(\Omega(v))$. In particular, $(\Omega(v), \alpha)$ has pure point dynamical spectrum. As every eigenvalue is simple, compare [69, Ch. 3] for the case of $\mathbb{Z}$-actions, this also shows that any character is continuous.

(iii) This is an application of the diffraction formula Eq. (5) in Sect. 3.3.

If $G$ is $\sigma$-compact, then both the autocorrelation and the diffraction measure can be computed via the usual limiting procedure.

Corollary 1 Assume that $G$ is $\sigma$-compact and fix any van Hove sequence $\left(B_{n}\right)_{n \in \mathbb{N}}$ in $G$. Let $v \in \mathcal{M}^{\infty}(G)$ be strongly almost periodic. Then, the following hold:

(i) The autocorrelation measure

$$
\gamma=\left.\lim _{n \rightarrow \infty} \frac{1}{m_{G}\left(B_{n}\right)} \omega\right|_{B_{n}} * \widetilde{\left.\omega\right|_{B_{n}}}
$$

exists as a vague limit for all $\omega \in \Omega(\nu)$, and the limit $\gamma \in \mathcal{M}^{\infty}(G)$ is independent of the choice of $\omega \in \Omega(v)$. The autocorrelation is a positive definite strongly almost periodic measure and hence Fourier transformable as a measure.

(ii) The diffraction measure $\widehat{\gamma} \in \mathcal{M}^{\infty}(\widehat{G})$ is pure point and given by

$$
\widehat{\gamma}=\sum_{\lambda \in \widehat{\Omega(v)}}\left|c_{\lambda \circ J}\right|^{2} \delta_{\lambda \circ J}
$$

In the above equation, we have $c_{\lambda \circ J}(\omega)=\lim _{n \rightarrow \infty} c_{\lambda \circ J}^{(n)}(\omega)$, where

$$
c_{\lambda \circ J}^{(n)}(\omega)=\frac{1}{m_{G}\left(B_{n}\right)} \int_{B_{n}}(\lambda \circ J)(t) \mathrm{d} \omega(t),
$$

and the limit $c_{\lambda \circ J}(\omega)$ has constant modulus, which we abbreviate by $\left|c_{\lambda_{\circ} \mathrm{J}}\right|$. 
Proof Existence of $\gamma$ and independence of the choice of $\omega \in \Omega(v)$ is discussed in Sect. 3.3. Pure point dynamical spectrum of $(\Omega(v), \alpha)$, which is shown in Theorem 1 , implies that $\widehat{\gamma}$ is a pure point measure, see, e.g., [60]. The explicit expression for the amplitudes follows from Eq. (4) in Sect. 3.3.

\section{Cut-and-project schemes and model sets}

As a preparation for the following sections, we explain the cut-and-project construction. In the physics community, it has been invented to obtain point sets with unusual symmetries [40] and diffraction [23] such as models of quasicrystals, see, e.g., [56] for a recent review. In fact that construction already emerged in Meyer's work [48] within a harmonic analysis context. Meyer's approach was later popularized by Moody. We will adopt his notation [50,51].

A triple $(G, H, \mathcal{L})$ is called a cut-and-project scheme (or simply CPS) if $G$ and $H$ are LCAG and $\mathcal{L}$ is a lattice in $G \times H$ (i.e., a co-compact discrete subgroup) such that

- the canonical projection $\pi^{G}: G \times H \longrightarrow G$ is one-to-one between $\mathcal{L}$ and $L:=\pi^{G}(\mathcal{L})$ (in other words, $\left.\mathcal{L} \cap(\{0\} \times H)=\{(0,0)\}\right)$, and

- the image $L^{\star}=\pi^{H}(\mathcal{L})$ of the canonical projection $\pi^{H}: G \times H \longrightarrow H$ is dense in $H$.

The group $H$ is called the internal space. Given these properties of the projections $\pi^{G}$ and $\pi^{H}$, one can define the so-called star map $(\cdot)^{\star}: L \longrightarrow H$ as follows. If $x \in L$, then there is a unique $y \in H$ such that $(x, y) \in \mathcal{L}$, and we set $x^{\star}=y$. If we denote the inverse map of $\left(\left.\pi^{G}\right|_{\mathcal{L}}\right)$ by $\left(\left.\pi^{G}\right|_{\mathcal{L}}\right)^{-1}: L \rightarrow \mathcal{L}$, we then have $x^{\star}=\left(\pi^{H} \circ\left(\left.\pi^{G}\right|_{\mathcal{L}}\right)^{-1}\right)(x)$. The situation is summarized in the following diagram.

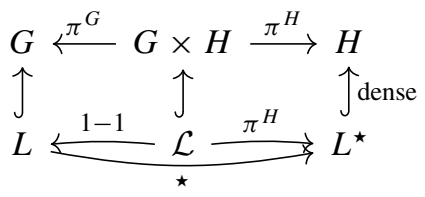

We fix Haar measures $m_{G}$ on $G$ and $m_{H}$ on $H$. We then denote by dens $(\mathcal{L})$ the inverse Haar measure of a measurable fundamental domain for $\mathcal{L}$. If $G \times H$ is $\sigma$-compact, it equals the density of lattice points in $G \times H$. Given a CPS, we can associate to any $W \subset H$, called the window, the set

$$
\text { 人 }(W):=\left\{x \in L: x^{\star} \in W\right\} .
$$

If $W$ is relatively compact, then $\curlywedge(W)$ is called a weak model set. Any weak model set is uniformly discrete. If in addition $\stackrel{\circ}{W} \neq \varnothing$, then $\curlywedge(W)$ is called a model set. Any model set is a Delone set, i.e., it is uniformly discrete and relatively dense.

A CPS gives rise to a dynamical system as follows. Define $\mathbb{T}:=(G \times H) / \mathcal{L}$. Then, $\mathbb{T}$ is a compact abelian group by assumption on $\mathcal{L}$. Let

$$
G \times H \longrightarrow \mathbb{T}, \quad(t, k) \mapsto[t, k],
$$


be the canonical quotient map. There is a canonical continuous group homomorphism

$$
\iota: G \longrightarrow \mathbb{T}, t \mapsto[t, 0]
$$

The homomorphism $\iota$ has dense range as $L^{\star}$ is dense in $H$. It induces an action $\beta$ of $G$ on $\mathbb{T}$ via

$$
\beta: G \times \mathbb{T} \longrightarrow \mathbb{T}, \beta_{t}([s, k]):=\iota(-t)+[s, k]=[s-t, k] .
$$

The dynamical system $(\mathbb{T}, \beta)$ will play a crucial role in our considerations as it appears in the torus parametrization of the hull associated with (a weighted version of) $\curlywedge(W)$, see Theorem 3 and $[38,45]$. The dynamical system $(\mathbb{T}, \beta)$ is minimal and uniquely ergodic, as $\iota$ has dense range. Moreover, it has pure point spectrum. More precisely, the dual group $\widehat{\mathbb{T}}$ gives a set of continuous eigenfunctions, which form a complete orthonormal basis by the Peter-Weyl theorem (see [61] and [60] for further details). By Pontryagin duality, the dual group $\widehat{\mathbb{T}}$ can be naturally identified with the dual lattice

$$
\mathcal{L}^{0}=\{(\chi, \eta) \in \widehat{G} \times \widehat{H}: \chi(x) \eta(y)=1 \text { for all }(x, y) \in \mathcal{L}\} .
$$

Here we identify $\lambda \in \widehat{\mathbb{T}}$ and $(\chi, \eta) \in \mathcal{L}^{0}$ if and only if $\lambda([t, k])=\chi(t) \eta(k)$ for all $[t, k] \in \mathbb{T}$. For a CPS $(G, H, \mathcal{L})$, the situation simplifies as $\left(\widehat{G}, \widehat{H}, \mathcal{L}^{0}\right)$ is also a CPS. As the projection $\left.\pi^{\widehat{G}}\right|_{\mathcal{L}^{0}}$ is one-to-one, we can write

$$
\mathcal{L}^{0}=\left\{\left(\chi, \chi^{\star}\right) \in \widehat{G} \times \widehat{H}: \chi(x) \chi^{\star}\left(x^{\star}\right)=1 \text { for all } x \in L\right\},
$$

and we may thus identify $\widehat{\mathbb{T}}$ with $L^{0}:=\pi^{\widehat{G}}\left(\mathcal{L}^{0}\right)$. The potential Bragg peak positions in the diffraction of $\curlywedge(W)$ are elements of $L^{0}$, compare Theorem 3 .

\section{Lattice modulations}

We now generalize the example of Sect. 2 to (almost periodic) modulations of lattices in LCAG, see "Appendix A" for basics about almost periodic functions taking values in a group. We will show that any modulated lattice is a deformed weighted lattice model set, and vice versa that any deformed weighted lattice model set is a modulated lattice. We will further show that the class of modulations of a lattice is stable under modulation. In Sect. 7 we will show that general deformed weighted model sets are strongly almost periodic. This means, in particular, that any lattice modulation is strongly almost periodic.

Definition 2 (modulated lattice) Let $L$ be lattice in a LCAG $G$, and let $w: L \rightarrow \mathbb{C}$ and $g: L \rightarrow G$ be almost periodic functions. Then, the weighted Dirac comb

$$
\delta_{L}^{w, g}=\sum_{\ell \in L} w(\ell) \delta_{\ell+g(\ell)}
$$

is called a modulation of $L$. The functions $w, g$ are called the modulation functions. 
Remark 2 (i) We interpret a lattice modulation as a measure. We thus allow for multiplicities, i.e., the above sum may include terms $\ell \neq \ell^{\prime}$ satisfying $\ell+g(\ell)=$ $\ell^{\prime}+g\left(\ell^{\prime}\right)$. The measure setting also allows to incorporate a weighting of the lattice points.

(ii) If the modulation functions are almost periodic, then the resulting lattice modulations are strongly almost periodic. This can be seen from Proposition 1 and Theorem 3. In order to analyze the converse statement, restrict to $G=\mathbb{R}$ and $w \equiv 1$. In that situation, the converse can be shown to be true if $g$ has sufficiently small range. However, the converse may not be true in general, as can be seen from the artificial example $g: \mathbb{Z} \rightarrow \mathbb{R}$ given by $g(0)=1, g(1)=-1$ and $g(n)=0$ otherwise.

(iii) As any modulation function is bounded, any modulation is a measure of locally finite support. Some geometric properties of modulated lattices are discussed in [25, Ex. 3.10].

\subsection{Lattice modulations and deformations}

We argue that modulated lattices are so-called deformed weighted model sets. Within that framework, we have a very good overview of structural properties of the modulated lattice. In fact, any deformed weighted lattice model set is a modulated lattice. Our arguments use the Bohr compactification of the lattice, see "Appendix A".

There is a natural choice for a CPS, which has compact internal space: The triple $\left(G, H_{L}, \mathcal{L}_{L}\right)$, where $H_{L}:=L_{\mathrm{b}}$ is the Bohr compactification of $L$, together with the lattice $\mathcal{L}_{L}=\left\{\left(\ell, i_{\mathrm{b}}(\ell)\right): \ell \in L\right\}$, where $i_{\mathrm{b}}: L \rightarrow L_{\mathrm{b}}$ is the canonical dense embedding. The lattice is the model set whose window is the whole internal space.

\section{Lemma 3 The above CPS is well-defined.}

Proof We argue that $\mathcal{L}_{L}$ is a lattice. It is clear that $\mathcal{L}_{L}$ is a group. To show uniform discreteness, take a zero neighborhood $U \subset G$ such that $L \cap U=\{0\}$. Then, $\mathcal{L}_{L} \cap(U \times$ $\left.H_{L}\right)=\{0\}$. Indeed, if $\left(\ell, i_{\mathrm{b}}(\ell)\right) \in U \times H_{L}$, then $\ell=0$, which implies that $i_{\mathrm{b}}(0)=0$ is the neutral element in $H_{L}$. Also, $\mathcal{L}_{L}$ is relatively dense in $G \times H_{L}$ as $L$ is relatively dense in $G$ and as $H_{L}$ is compact. Thus, $\mathcal{L}_{L}$ is a lattice in $G \times H_{L}$. By construction, $\pi^{H_{L}}\left(\mathcal{L}_{L}\right)=i_{\mathrm{b}}(L)$ is dense in $H_{L}$. To see that $\pi^{G}$ is one-to-one on $\mathcal{L}_{L}$, consider any $\left(\ell_{1}, i_{\mathrm{b}}\left(\ell_{1}\right)\right)$ and $\left(\ell_{2}, i_{\mathrm{b}}\left(\ell_{2}\right)\right)$ such that $\ell_{1}=\ell_{2}$. Then, also $i_{\mathrm{b}}\left(\ell_{1}\right)=i_{\mathrm{b}}\left(\ell_{2}\right)$.

We next explain the notion of deformed weighted model set. Whereas the general definition will be analyzed from Sect. 7 onward, in this section we will consider the special case that the underlying model set is a lattice.

Definition 3 (Deformed weighted model set) Let $(G, H, \mathcal{L}$ ) be any CPS. Let $f \in$ $C_{c}(H)$ and $p \in C(H, G)$ be weight and deformation functions and let $L=\pi^{G}(\mathcal{L})$.

(i) The weighted Dirac comb

$$
v=\sum_{\ell \in L} f\left(\ell^{\star}\right) \delta_{\ell+p\left(\ell^{\star}\right)}
$$


is called a deformed weighted model set in the CPS $(G, H, \mathcal{L})$.

(ii) If $H$ is compact, then $L$ is a lattice in $G$, as $L=\pi^{G}(\mathcal{L} \cap(G \times H))$ is both a model set and a group. In that case we speak of a deformed weighted lattice model set.

The following three results show that the class of deformed weighted lattice model sets coincides with the class of lattice modulations.

Proposition 1 Any lattice modulation is a deformed weighted lattice model set in the CPS of Lemma 3.

Proof Use the Bohr compactification $L_{\mathrm{b}}$ to write $w=w_{\mathrm{b}} \circ i_{\mathrm{b}}$ for $w_{\mathrm{b}} \in C\left(L_{\mathrm{b}}\right)$ and $g=g_{\mathrm{b}} \circ i_{\mathrm{b}}$ for $g_{\mathrm{b}} \in C\left(L_{\mathrm{b}}, G\right)$. Define $f \in C_{c}\left(H_{L}\right)$ by $f=w_{\mathrm{b}}$, and define $p \in C\left(H_{L}, G\right)$ by $p=g_{\mathrm{b}}$. We then have

$$
\sum_{\ell \in L} f\left(\ell^{\star}\right) \delta_{\ell+p\left(\ell^{\star}\right)}=\sum_{\ell \in L} w_{\mathrm{b}}\left(i_{\mathrm{b}}(\ell)\right) \delta_{\ell+g_{\mathrm{b}}\left(i_{\mathrm{b}}(\ell)\right)}=\sum_{\ell \in L} w(\ell) \delta_{\ell+g(\ell)} .
$$

Remark 3 The previous proposition states that any modulated lattice is a deformed weighted model set. As a consequence, any modulated lattice is strongly almost periodic by Theorem 3 .

The above CPS is, in some sense, a universal CPS scheme for the lattice $L$. This idea is further elaborated in Sect. 8. Here, we note the following result.

Proposition 2 Let $(G, H, \mathcal{L})$ be a CPS with compact $H$ and lattice $L=\pi^{G}(\mathcal{L})$. Then, any deformed weighted lattice model set in $(G, H, \mathcal{L})$ is a deformed weighted lattice model set in $\left(G, H_{L}, \mathcal{L}_{L}\right)$.

Proof Let $f \in C_{c}(H)$ be the weight function and let $p \in C(H, G)$ be the deformation map. Since $L=\curlywedge(H)$, the star map ${ }^{\star}: L \rightarrow H$ is a continuous homomorphism from $L$ to the compact group $H$. By the universal property of the Bohr compactification, we may find a continuous group homomorphism $\psi: L_{\mathrm{b}}=H_{L} \rightarrow H$ such that for all $\ell \in L$ we have $\psi\left(i_{b}(\ell)\right)=\ell^{\star}$. Then, $f^{\prime}=f \circ \psi \in C_{c}\left(H_{L}\right)$ and $p^{\prime}=p \circ \psi \in$ $C\left(H_{L}, G\right)$, and in the CPS $\left(G, H_{L}, \mathcal{L}_{L}\right)$ we have

$$
\sum_{\ell \in L} f^{\prime}\left(i_{\mathrm{b}}(\ell)\right) \delta_{\ell+p^{\prime}\left(i_{\mathrm{b}}(\ell)\right)}=\sum_{\ell \in L} f\left(\psi\left(i_{\mathrm{b}}(\ell)\right)\right) \delta_{\ell+p\left(\psi\left(i_{\mathrm{b}}(\ell)\right)\right)}=\sum_{\ell \in L} f\left(\ell^{\star}\right) \delta_{\ell+p\left(\ell^{\star}\right)} .
$$

Proposition 3 Any deformed lattice model set in $\left(G, H_{L}, \mathcal{L}_{L}\right)$ is a modulation of $L$.

Proof Assume that the deformed weighted lattice model set has weight function $f \in$ $C_{c}\left(H_{L}\right)$ and deformation function $p \in C\left(H_{L}, G\right)$. Then, the deformed weighted lattice model set can be written as $v=\sum_{\ell \in L} f\left(\ell^{\star}\right) \delta_{\ell+p\left(\ell^{\star}\right)}$. Now $f\left(\ell^{\star}\right)=\left(f \circ i_{\mathrm{b}}\right)(\ell)$, and $f \circ i_{\mathrm{b}}: L \rightarrow \mathbb{C}$ is almost periodic by Proposition 9. Also $p\left(\ell^{\star}\right)=\left(p \circ i_{\mathrm{b}}\right)(\ell)$, and $p \circ i_{\mathrm{b}}: L \rightarrow G$ is almost periodic by Proposition 9 . Hence, $v$ is a modulation of $L$ with modulation functions $w=f \circ i_{\mathrm{b}}$ and $g=p \circ i_{\mathrm{b}}$. 
We gather the previous results in the following theorem.

Theorem 2 The class of modulated lattices coincides with the class of deformed weighted lattice model sets.

Remark 4 The above general results have been obtained using the Bohr compactification of the lattice. However, when analyzing particular examples, one might construct a "minimal" internal space using the modulation function, see the following example. This is in line with the experimental situation, where the number of Miller indices is determined and a torus of appropriate dimension is added to an underlying crystallographic model. This approach has been phrased in terms of model sets in [63, Sec. 3].

Example 1 (Sine modulated integers revisited) Consider the situation of a Dirac comb $\sum_{\ell \in L} \delta_{\ell+g(\ell)}$, i.e., the modulation function $w$ is constant. We will use the group compactification $L_{g}=\overline{\left\{T_{\ell} g^{\dagger}: \ell \in L\right\}}$ of $L$ by $g^{\dagger}$, where $g^{\dagger}(x)=g(-x)$. Noting that $\mathcal{L}_{g}=\left\{\left(\ell, T_{\ell} g^{\dagger}\right): \ell \in L\right\}$ is a lattice in $G \times L_{g}$, we can consider the CPS $\left(G, L_{g}, \mathcal{L}_{g}\right)$. Define $f \in C_{c}\left(L_{g}\right)$ by $f \equiv 1$ and $p \in C\left(L_{g}, G\right)$ by $p(h)=h(0)$ for $h \in C\left(L_{g}, G\right)$. We then have

$$
\sum_{\ell \in L} f\left(\ell^{\star}\right) \delta_{\ell+p\left(\ell^{\star}\right)}=\sum_{\ell \in L} \delta_{\ell+g(\ell)},
$$

i.e., the modulated lattice is a deformed weighted lattice model set in the CPS $\left(G, L_{g}, \mathcal{L}_{g}\right)$. This construction was used for sine modulated integers in Sect. 2.2.

\subsection{Modulations of modulated lattices}

We show that the class of modulations of a lattice is stable under modulation. We first extend the definition of modulation beyond the lattice case.

Definition 4 (modulated measure) Given $v \in \mathcal{M}(G)$, let $w \in C_{\mathrm{u}}(G)$ and $g \in$ $C_{\mathrm{u}}(G, G)$ be almost periodic functions. Then, the measure $v^{w, g} \in \mathcal{M}(G)$, defined by

$$
v^{w, g}(\varphi)=\int w(x) \varphi(x+g(x)) \mathrm{d} v(x)
$$

for $\varphi \in C_{c}(G)$, is called a modulation of $v$. The functions $w, g$ are called the modulation functions.

Remark 5 The above indeed extends Definition 2 of a modulated lattice. The domain of the modulation functions is chosen to be the group $G$. Almost periodicity is inherited by passing to subgroups, compare Lemma 8.

Proposition 4 (lattice modulations are modulation stable) Let $\delta_{L}^{w, g}$ be a modulation of $L$, and let $w^{\prime} \in C_{u}(G)$ and $g^{\prime} \in C_{u}(G, G)$ be almost periodic functions. Then, $\left(\delta_{L}^{w, g}\right)^{w^{\prime}, g^{\prime}}$ is a modulation of $L$. 
Proof Note first that by definition we have

$$
\left(\delta_{L}^{w, g}\right)^{w^{\prime}, g^{\prime}}=\left(\sum_{\ell \in L} w(\ell) \delta_{\ell+g(\ell)}\right)^{w^{\prime}, g^{\prime}}=\sum_{\ell \in L} w(\ell) w^{\prime}(\ell+w(\ell)) \delta_{\ell+g(\ell)+g^{\prime}(\ell+g(\ell))} .
$$

The statement follows if $g^{\prime \prime} \in C_{\mathrm{u}}(L, G)$, given by $g^{\prime \prime}(\ell)=g(\ell)+g^{\prime}(\ell+g(\ell))$, and $w^{\prime \prime} \in C_{\mathrm{u}}(L)$, given by $w^{\prime \prime}(\ell)=w(\ell)+w^{\prime}(\ell+w(\ell))$, are almost periodic on $L$. We show this for $g^{\prime \prime}$, the argument for $w^{\prime \prime}$ is analogous. Write $g=g_{\mathrm{b}} \circ i_{L}$ for $g_{\mathrm{b}} \in C\left(L_{\mathrm{b}}, G\right)$ and $g^{\prime}=g_{\mathrm{b}}^{\prime} \circ i_{G}$ for $g_{\mathrm{b}}^{\prime} \in C\left(G_{\mathrm{b}}, G\right)$. Using the notation of Lemma 7 , we have

$$
\begin{aligned}
g^{\prime \prime}(\ell) & =g(\ell)+g^{\prime}(\ell+g(\ell))=g_{\mathrm{b}} \circ i_{L}(\ell)+g_{\mathrm{b}}^{\prime} \circ i_{G}\left(\ell+g_{\mathrm{b}} \circ i_{L}(\ell)\right) \\
& =g_{\mathrm{b}} \circ i_{L}(\ell)+g_{\mathrm{b}}^{\prime}\left(i_{\mathrm{b}} \circ i_{L}(\ell)+i_{G} \circ g_{\mathrm{b}} \circ i_{L}(\ell)\right) .
\end{aligned}
$$

Together with Lemma 7 it follows that $g^{\prime \prime}=g_{\mathrm{b}}^{\prime \prime} \circ i_{L}$ for some $g_{\mathrm{b}}^{\prime \prime} \in C\left(L_{\mathrm{b}}, G\right)$. Hence, $g^{\prime \prime}$ is almost periodic on $L$ by Proposition 9 .

\section{Deformed weighted model sets}

We give a large class of strongly almost periodic measures arising from model sets. In general, Dirac combs of model sets are not strongly almost periodic. However, weighted model sets [7] and more general dense Dirac combs [45] are strongly almost periodic. Here we show that also weighted versions of deformed model sets $[8,13]$ are strongly almost periodic. This comprises the weighted model sets with continuous weight functions of $[11,45,58,65]$. Also ideal crystals and their modulations, which are discussed in Sect. 9, fall into this class.

Theorem 3 Consider the setting of Definition 3 (i). Then, the following hold.

(i) The deformed weighted model set $v \in \mathcal{M}^{\infty}(G)$ is strongly almost periodic.

(ii) The hull $\Omega(v)$ is a compact abelian group, and the canonical action $\alpha$ is continuous and onto. In particular, $(\Omega(v), \alpha)$ is minimal, uniquely ergodic with continuous eigenfunctions. The unique ergodic measure is the Haar measure on the group, and the set of continuous eigenfunctions is the group dual to $\Omega(v)$.

(iii) Consider $\mathbb{T}=(G \times H) / \mathcal{L}$ together with canonical $G$-action $\beta$. The topological dynamical system $(\Omega(\nu), \alpha)$ is a factor of $(\mathbb{T}, \beta)$ with factor map $\mu: \mathbb{T} \rightarrow \Omega(v)$ given by

$$
\mu([s, k])(\varphi)=\sum_{\ell \in L} f\left(\ell^{\star}+k\right) \varphi\left(\ell+s+p\left(\ell^{\star}+k\right)\right)
$$

for any $\varphi \in C_{c}(G)$. In fact $\mu$ is a group homomorphism.

(iv) The diffraction measure of any $\omega \in \Omega(v)$ is given by $\widehat{\gamma}=\sum_{\chi \in L^{0}}\left|a_{\chi}\right|^{2} \delta_{\chi}$, where

$$
a_{\chi}=\operatorname{dens}(\mathcal{L}) \cdot \int_{H} \overline{\chi^{\star}(y)} \chi(p(y)) f(y) \mathrm{d} m_{H}(y) .
$$


Here dens $(\mathcal{L})$ is the inverse measure of a measurable fundamental domain for the lattice $\mathcal{L}$. In the $\sigma$-compact case, it coincides with the density of lattice points in $G \times H$.

Remark 6 (i) The continuous map $\mu: \mathbb{T} \rightarrow \Omega(v)$ may be called the torus parametrization of $\Omega(v)$. For undeformed weighted model sets, it has previously been used in [45].

(ii) Consider (unweighted and undeformed) model set Dirac combs. In that case, continuity of $\mu$ fails in general. A torus parametrization is then often defined as a natural continuous map $\Omega(v) \rightarrow \mathbb{T}$, which is a partial inverse of $\mu$. On the other hand, $\mu$ is measurable in that case, which is sufficient in order to infer diffraction properties [38,39].

(iii) The proofs of parts (i), (ii), (iii) adapt arguments from [45, Sec. 7]. With more technical effort, the above setting could be extended to comprise deformations of dense Dirac combs [45].

(iv) The formula for the diffraction measure in ( $i v)$ shows that in comparison to the undeformed model set, the potential Bragg peak positions are unchanged, but their amplitudes are altered. In the Euclidean setting, the above result has been obtained in [13], by calculating the autocorrelation of the deformed weighted model set and its transform using Eq. (4). We will give a proof based on almost periodicity and dynamical systems, which uses Eq. (5).

(v) If there are no extinctions (i.e., zero amplitudes at potential Bragg peak positions), then the result discussed in (iv) says that both hulls are spectrally isomorphic. In that case both hulls are also measure-theoretically isomorphic, by the Halmos-von Neumann theorem (see, e.g., [69] for the case of $\mathbb{Z}$-actions). Hence, dynamical concepts stronger than measure-theoretic isomorphism are needed in order to distinguish the different types of diffraction.

(vi) The statements in (iii) and (iv) are also proved in [8, Thm. 6] and in [44, Sec. 7.1] by a different method. Diffraction calculations for deformed (unweighted) model sets also appear in $[8,13]$. In particular, there are explicit calculations for the Fibonacci chain [13, Sec. 5] and the silver mean chain [8, Sec. 7].

Proof (i) We show that $v$ is strongly almost periodic. This implies translation boundedness, compare Sect. 4. Consider any $\varphi \in C_{c}(G)$. Note first that for any $y \in G$ and for any $t \in L$ we have by a standard estimate

$$
\begin{gathered}
|(v * \varphi)(y)-(v * \varphi)(y-t)| \leq \sum_{\ell \in L} \mid f\left(\ell^{\star}\right) \varphi\left(y-\ell-p\left(\ell^{\star}\right)\right)-f\left(\ell^{\star}\right. \\
\left.-t^{\star}\right) \varphi\left(y-\ell-p\left(\ell^{\star}-t^{\star}\right)\right) \mid .
\end{gathered}
$$

Now take any compact zero neighborhood $U$ in $H$ and restrict to $t \in L$ such that $t^{\star} \in U$, i.e., restrict to $t \in \curlywedge(U)$. Then, we may have non-vanishing summands only if $\ell^{\star} \in \operatorname{supp}(f) \cup(\operatorname{supp}(f)+U)=: W$, which is a compact set. In addition to the previous condition, we may have non-vanishing summands only if $\ell \in y-p(W)-\operatorname{supp}(\varphi)=$ : $y+K$, which is a compact set. Hence, the above sum may be restricted without loss of generality to $\ell \in \curlywedge(W) \cap(y+K)$, which is a finite set. Using the triangle inequality 
and standard estimates, we thus get for any $t \in \mathcal{\curlywedge}(U)$ the estimate

$$
\begin{aligned}
& |(v * \varphi)(y)-(\nu * \varphi)(y-t)| \\
& \leq \sum_{\ell \in \curlywedge}\left|f\left(\ell^{\star}\right)-f\left(\ell^{\star}-t^{\star}\right)\right|\|\varphi\|_{\infty} \\
& \quad+\sum_{\ell \in \curlywedge(W) \cap(y+K)}\|f\|_{\infty}\left|\varphi\left(y-\ell-p\left(\ell^{\star}\right)\right)-\varphi\left(y-\ell-p\left(\ell^{\star}-t^{\star}\right)\right)\right| .
\end{aligned}
$$

Now consider the first sum in the above estimate. As $\curlywedge(W)$ is uniformly discrete, the number of terms in the sum is bounded uniformly in $y \in G$. As $f$ is uniformly continuous, this implies that the first sum gets arbitrarily small if $t^{\star}$ approaches zero, uniformly in $y \in G$. We can argue similarly for the second sum, using that $\varphi$ is uniformly continuous and that $p$ is uniformly continuous when restricted to the compact set $W$. Hence, if arbitrary $\varepsilon>0$ is given, we find a zero neighborhood $V \subset U$ such that

$$
|(v * \varphi)(y)-(v * \varphi)(y-t)| \leq \varepsilon
$$

for all $y \in G$ and for every $t \in \mathcal{\lambda}(V)$. As $\curlywedge(V)$ is relatively dense and as $\varphi$ was arbitrary, this shows that $v$ is strongly almost periodic.

(ii) This follows from (i) and Theorem 1.

(iii) We first show that $\mu: \mathbb{T} \rightarrow \Omega(v)$ is well-defined and continuous. Consider the map $\mu^{\prime}(s, k): C_{c}(G) \rightarrow \mathbb{C}$, given by

$$
\varphi \mapsto \mu^{\prime}(s, k)=\sum_{\ell \in L} f\left(\ell^{\star}+k\right) \varphi\left(\ell+s+p\left(\ell^{\star}+k\right)\right) .
$$

As $\mu^{\prime}$ is $\mathcal{L}$-invariant, the map $\mu$ is well-defined. The map $\mu^{\prime}$ is continuous: Let $\left(\xi_{\iota}\right)_{\iota \in I}$ be any net in $G \times H$ such that $\xi_{\iota} \rightarrow(s, k)$. Writing $\xi_{\iota}=\left(s_{\iota}, k_{l}\right)$, we have $s_{\iota} \rightarrow s$ and $k_{\iota} \rightarrow k$ as the canonical projections are continuous. We show $\mu^{\prime}\left(s_{\iota}, k_{\iota}\right)(\varphi) \rightarrow \mu^{\prime}(s, k)$. As the factor map is continuous, this implies continuity of $\mu$. Choose a compact neighborhood $W_{k} \subset H$ of $\operatorname{supp}(f)-k$ and choose a compact neighborhood $K_{s} \subset G$ of $\operatorname{supp}(\varphi)-p(\operatorname{supp}(f))-s$. Then, there is $\iota_{0} \in I$ such that $\operatorname{supp}(f)-k_{\iota} \subset W_{k}$ and $\operatorname{supp}(\varphi)-p(\operatorname{supp}(f))-s_{\iota} \subset K_{s}$ for all $\iota>\iota_{0}$. Using the triangle inequality and standard estimates, we obtain for $\iota>\iota_{0}$ the estimate

$$
\begin{aligned}
& \left|\mu^{\prime}(s, k)(\varphi)-\mu^{\prime}\left(s_{\iota}, k_{\iota}\right)(\varphi)\right| \\
& \leq \sum_{\ell \in \curlywedge\left(W_{k}\right) \cap K_{s}}\left|f\left(\ell^{\star}+k\right)-f\left(\ell^{\star}+k_{\iota}\right)\right|\|\varphi\|_{\infty} \\
& \quad+\sum_{\ell \in \curlywedge\left(W_{k}\right) \cap K_{s}}\|f\|_{\infty}\left|\varphi\left(\ell+s+p\left(\ell^{\star}+k\right)\right)-\varphi\left(\ell+s_{\iota}+p\left(\ell^{\star}+k_{\iota}\right)\right)\right| .
\end{aligned}
$$


As the above sums are finite, the rhs tends to zero with increasing $\iota$ as $f, \varphi$ and $p$ are continuous. This shows continuity of $\mu^{\prime}$ and of $\mu$, as the projection map is continuous. By definition, we have $\alpha_{t}(\mu([s, k]))=\mu\left(\beta_{t}([s, k])\right)$ for every $t \in G$ and every $(s, k) \in G \times H$. In particular, we have

$$
\mu\left(\beta_{t}([0,0])\right)=\alpha_{t}(\mu([0,0]))=\alpha_{t}\left(\mu^{\prime}(0,0)\right)=\alpha_{t} \nu .
$$

By minimality of $\mathbb{T}$, continuity of $\mu$ and compactness of $\mathbb{T}$ we thus have

$$
\mu(\mathbb{T})=\mu\left(\overline{\left\{\beta_{t}([0,0]): t \in G\right\}}\right)=\overline{\left\{\alpha_{t} \nu: t \in G\right\}}=\Omega(\nu) .
$$

We have shown that $\mu: \mathbb{T} \rightarrow \Omega(v)$ is continuous, onto and commutes with the translation action. Hence, $\mu$ is a factor map. Let the group homomorphism $J: G \longrightarrow$ $\Omega(v), t \mapsto \alpha_{t} \nu$ be given by Lemma 2. Then, Eq. (6) gives that $J(t)=\alpha_{t} v=\mu(\iota(t))$. Using Lemma 2, we thus have

$$
\mu(\iota(t) \dot{+} x)=J(t) \dot{+} \mu(x)=\mu(\iota(t)) \dot{+} \mu(x)
$$

for all $t \in G$ and $x \in \mathbb{T}$. Thus $\mu$ is a group homomorphism by minimality.

(iv) By (iii), the diffraction measure $\widehat{\gamma}$ of $v$ is pure point. As the torus parametrization map $\mu: \mathbb{T} \rightarrow \Omega(v)$ is a factor map, it can be used to embed $L^{2}(\Omega(v))$ isometrically into $L^{2}(\mathbb{T})$, compare [8, Thm. 1]. Hence, we can compute the diffraction using Eq. (5). The normalized $L^{2}(\mathbb{T})$-eigenfunctions of the translation $\beta$ are given by $e_{\chi}=\left(\chi, \chi^{\star}\right) \in$ $\widehat{\mathbb{T}} \sim \mathcal{L}^{0}$ and can be parametrized by their eigenvalue $\chi \in L^{0}$. Now by an application of Weil's formula [55, Eqn. (3.3.10)], also called the quotient integral formula [22, Thm. 1.5.2], we get

$$
\begin{aligned}
a_{\chi} & =\int_{\mathbb{T}} \overline{e_{\chi}([t, k])} \mu([t, k])(\chi \cdot \varphi) \mathrm{d}([t, k]) \\
& =\operatorname{dens}(\mathcal{L}) \cdot \int_{G} \int_{H} \overline{e_{\chi}(x, y)} f(y) \chi(x+p(y)) \varphi(x+p(y)) \mathrm{d}(x, y) \\
& =\operatorname{dens}(\mathcal{L}) \cdot \int_{H} \chi(p(y)) \overline{\chi^{\star}(y)} f(y) \mathrm{d} y .
\end{aligned}
$$

The factor dens $(\mathcal{L})$ reflects the non-canonical choice of Haar measure on $\mathbb{T}$.

\section{Modulations of deformed weighted model sets}

In this section, we will prove that any modulation of a deformed weighted model set is a deformed weighted model set. We will also prove that in Euclidean space, the class of deformed weighted model sets coincides with the class of modulated weighted model sets. 


\subsection{An extended CPS for modulations}

Let $\left(G, H_{0}, \mathcal{L}_{0}\right)$ be any CPS. The Bohr compactification of $G$ can be used to expand the internal space $H_{0}$. In the resulting CPS $(G, H, \mathcal{L})$, the lattice projects injectively to $H$. We will use that scheme in order to describe modulations of a deformed weighted model set.

Lemma 4 (Extended CPS) Let $\left(G, H_{0}, \mathcal{L}_{0}\right)$ be any $C P S$. Then, there exists an extended $C P S(G, H, \mathcal{L})$ where $\left.\pi^{H}\right|_{\mathcal{L}}$ is one-to-one with the following property: Every \{weak, deformed, weighted model set in $\left(G, H_{0}, \mathcal{L}_{0}\right)$ is a \{weak, deformed, weighted model set in $(G, H, \mathcal{L})$.

Proof Consider the Bohr compactification $G_{\mathrm{b}}$ of $G$ together with the completion homomorphism $i_{\mathrm{b}}: G \rightarrow G_{\mathrm{b}}$. Note that $i_{\mathrm{b}}$ is one-to-one, since $G$ is abelian. Setting $H^{\prime}=H_{0} \times G_{\mathrm{b}}$, we extend the star map ${ }^{\star} H_{0}$ from $H_{0}$ to $H^{\prime}$ by defining $\ell^{\star}=$ $\left(\ell^{\star} \mid H_{0}, i_{\mathrm{b}}(\ell)\right)$ for $\ell \in \pi^{G}\left(\mathcal{L}_{0}\right)=L_{0}$. We claim that the subgroup $\mathcal{L}=\left\{\left(\ell, \ell^{\star}\right): \ell \in\right.$ $\left.L_{0}\right\}$ of $G \times H^{\prime}$ is a lattice. To show discreteness of $\mathcal{L}$ in $G \times H^{\prime}$, choose open $U_{0} \subset$ $G \times H_{0}$ such that $\mathcal{L}_{0} \cap U_{0}=\{0\}$, define $U=U_{0} \times G_{\mathrm{b}}$ and consider $\left(\ell, \ell^{\star}\right) \in \mathcal{L} \cap U$. Then, $\ell=0$ due to discreteness of $\mathcal{L}_{0}$, which implies $\ell^{\star}=0$. Since $U$ is non-empty open, it follows that $\mathcal{L}$ is discrete. But $\mathcal{L}$ is also relatively dense in $G \times H^{\prime}$. To see this, take compact $K_{0} \subset G \times H_{0}$ such that $\mathcal{L}+K_{0}=G \times H_{0}$. Then, $K_{0} \times G_{\mathrm{b}}$ is compact, and we have

$$
\mathcal{L}+\left(K_{0} \times G_{\mathrm{b}}\right)=\left(\mathcal{L}_{0}+K_{0}\right) \times G_{\mathrm{b}}=G \times H^{\prime} .
$$

Injectivity of $\left.\pi^{G}\right|_{\mathcal{L}}$ is inherited from injectivity of $\left.\pi^{G}\right|_{\mathcal{L}_{0}}$. If we now define $H=$ $\overline{\pi^{H^{\prime}}(\mathcal{L})} \subset H^{\prime}$, the lattice $\mathcal{L}$ projects densely to $H$ by definition. Hence, $(G, H, \mathcal{L})$ is a CPS. The above formulae can also be used to infer injectivity of $\pi^{H}$ on $\mathcal{L}$ from injectivity of $i_{\mathrm{b}}$.

Now let $\curlywedge\left(W_{0}\right)$ be a weak model set in $\left(G, H_{0}, \mathcal{L}_{0}\right)$. Note that $W=W_{0} \times G_{\mathrm{b}}$ is relatively compact and that

$$
\begin{aligned}
\curlywedge\left(W_{0}\right) & =\left\{\pi^{G}\left(z_{0}\right) \mid z_{0} \in \mathcal{L}_{0}, \pi^{H_{0}}\left(z_{0}\right) \in W_{0}\right\} \\
& =\left\{\pi^{G}(z) \mid z \in \mathcal{L},\left(\pi^{H_{0}}(z), i_{\mathrm{b}}(z)\right) \in W_{0} \times G_{\mathrm{b}}\right\}=\curlywedge(W) .
\end{aligned}
$$

Assume that $v=\sum_{\ell \in L_{0}} f_{0}\left(\ell^{\star \mid} H_{0}\right) \delta_{\ell+p_{0}\left(\ell^{\star \mid} H_{0}\right)}$ is a deformed weighted model set in $\left(G, H_{0}, \mathcal{L}_{0}\right)$, where $f_{0} \in C_{c}\left(H_{0}\right)$ and $p_{0} \in C\left(H_{0}, G\right)$. Define $f \in C_{c}(H)$ by $f(y)=$ $f_{0}\left(\pi^{H_{0}}(y)\right)$ and $p \in C(H, G)$ by $p(y)=p_{0}\left(\pi^{H_{0}}(y)\right)$. As we have $L_{0}=L=\pi^{G}(\mathcal{L})$, we conclude $v=\sum_{\ell \in L} f\left(\ell^{\star}\right) \delta_{\ell+p\left(\ell^{\star}\right)}$ is a deformed weighted model set in $(G, H, \mathcal{L})$.

\subsection{Modulations of deformed weighted model sets}

The class of deformed weighted model sets is stable under modulations. The argument is analogous to that of Proposition 4. 
Theorem 4 (Deformed weighted model sets are modulation stable) Let $(G, H, \mathcal{L})$ be an extended CPS as in the previous subsection. Then, any modulation of a deformed weighted model set in $(G, H, \mathcal{L})$ is a deformed weighted model set in $(G, H, \mathcal{L})$.

Proof Consider $H \subset H^{\prime}=H_{0} \times G_{\mathrm{b}}$, where we use the notation of the previous subsection. Write $L=\pi^{G}(\mathcal{L})$. Let $\sum_{\ell \in L} f\left(\ell^{\star}\right) \delta_{\ell+p\left(\ell^{\star}\right)}$ be any deformed weighted model set in $(G, H, \mathcal{L})$, where $f \in C_{c}(H)$ and where $p \in C(H, G)$. Let $w \in C_{\mathrm{u}}(G)$ and $g \in C_{\mathrm{u}}(G, G)$ be almost periodic functions for the modulation. Choose $g_{\mathrm{b}} \in$ $C\left(G_{\mathrm{b}}, G\right)$ such that $g=g_{\mathrm{b}} \circ i_{\mathrm{b}}$. We then have for $\ell \in L$ the equality

$$
\begin{aligned}
p\left(\ell^{\star}\right)+g\left(\ell+p\left(\ell^{\star}\right)\right) & \left.=p\left(\ell^{\star}\right)+\left(g_{\mathrm{b}} \circ i_{\mathrm{b}}\right)\left(\ell+p\left(\ell^{\star}\right)\right)\right) \\
& =p\left(\ell^{\star}\right)+g_{\mathrm{b}}\left(\pi^{G_{\mathrm{b}}}\left(\ell^{\star}\right)+i_{\mathrm{b}}\left(p\left(\ell^{\star}\right)\right)\right) .
\end{aligned}
$$

We can now use the rhs of the above equation to define $p^{\prime} \in C(H, G)$ by $p^{\prime}(y)=$ $p(y)+g_{\mathrm{b}}\left(\pi^{G_{\mathrm{b}}}(y)+i_{\mathrm{b}}(p(y))\right)$. Similarly, we choose $w_{\mathrm{b}} \in C\left(G_{\mathrm{b}}\right)$ such that $w=$ $w_{\mathrm{b}} \circ i_{\mathrm{b}}$, and we get

$$
\left.w\left(\ell+p\left(\ell^{\star}\right)\right)=\left(w_{\mathrm{b}} \circ i_{\mathrm{b}}\right)\left(\ell+p\left(\ell^{\star}\right)\right)\right)=w_{\mathrm{b}}\left(\pi^{G_{\mathrm{b}}}\left(\ell^{\star}\right)+i_{\mathrm{b}}\left(p\left(\ell^{\star}\right)\right)\right) .
$$

Let us define $f^{\prime} \in C_{c}(H)$ by $f^{\prime}(y)=f(y) \cdot w_{\mathrm{b}}\left(\pi^{G_{\mathrm{b}}}(y)+i_{\mathrm{b}}(p(y))\right)$. We then have

$$
\sum_{\ell \in L} w\left(\ell+p\left(\ell^{\star}\right)\right) f\left(\ell^{\star}\right) \delta_{\ell+p\left(\ell^{\star}\right)+g\left(\ell+p\left(\ell^{\star}\right)\right)}=\sum_{\ell \in L} f^{\prime}\left(\ell^{\star}\right) \delta_{\ell+p^{\prime}\left(\ell^{\star}\right)} .
$$

\subsection{Modulations and deformations}

Specializing Theorem 4 to the case $p \equiv 0$, we get that modulations of weighted model sets are deformed weighted model sets.

Corollary 2 Let $\left(G, H_{0}, \mathcal{L}_{0}\right)$ be any CPS, and let $(G, H, \mathcal{L})$ be the extended CPS of Lemma 4, where $H \subset H_{0} \times G_{b}$. Then, for any two almost periodic functions $w \in C_{u}(G)$ and $g \in C_{u}(G, G)$ there exist $f \in C_{c}(H)$ and $p \in C(H, G)$ such that

$$
w(\ell)=f\left(\ell^{\star}\right), \quad g(\ell)=p\left(\ell^{\star}\right)
$$

for all $\ell \in \pi^{G}(\mathcal{L})$. In particular, any modulation of a weighted model set in $\left(G, H_{0}, \mathcal{L}_{0}\right)$ is a deformed weighted model set in $(G, H, \mathcal{L})$.

The following result states that for $G=\mathbb{R}^{d}$ the converse also holds. Hence, in Euclidean space the class of modulated weighted model sets coincides with the class of deformed weighted model sets.

Proposition 5 Let $(G, H, \mathcal{L})$ be any $C P S$ for $G=\mathbb{R}^{d}$. Consider $f \in C_{c}(H)$ and $p \in$ $C(H, G)$. Then, there exist almost periodic functions $w \in C_{u}(G)$ and $g \in C_{u}(G, G)$ 
such that

$$
w(\ell)=f\left(\ell^{\star}\right), \quad g(\ell)=p\left(\ell^{\star}\right)
$$

for all $\ell \in \hat{\curlywedge}(W)$ where $W=\operatorname{supp}(f)$. In particular, any deformed weighted model set in $(G, H, \mathcal{L})$ is a modulation of a weighted model set in $(G, H, \mathcal{L})$.

Proof We construct $g$ by exploiting the $\mathbb{R}$-vector space structure of $\mathbb{R}^{d}$. We may assume without loss of generality that $p: H \rightarrow G$ is constant 0 outside a precompact neighborhood $W_{0}$ of $W$. Indeed, pick some $h \in C_{c}(H)$ such that $h \equiv 1$ on $W$, and define $p^{\prime}: H \rightarrow G$ via $p^{\prime}(y)=h(y) \cdot p(y)$. Then, $p$ vanishes outside the precompact set $W_{0}=\{y \in H: h(y) \neq 0\}$, and we can replace $p$ by $p^{\prime}$. In particular, $p$ can be assumed to be uniformly continuous without loss of generality.

Next, take any zero precompact neighborhood $V_{0} \subset H$ and take any zero neighborhood $U_{0}$ in $G$ such that the regular model set $\curlywedge\left(W_{0}+V_{0}\right)$ is $U_{0}$-uniformly discrete. This is possible as $W_{0}+V_{0}$ is precompact, and hence, $\curlywedge\left(W_{0}+V_{0}\right)$ is uniformly discrete.

Finally, choose $\varphi \in C_{c}(G)$ such that $\varphi(\mathbf{0})=1,0 \leq \varphi \leq 1$ and $\operatorname{supp}(\varphi) \subset U_{0}$ and define $g: G \rightarrow G$ by

$$
g(x)=\sum_{\ell \in \curlywedge} \varphi(x-\ell) \cdot p\left(\ell^{\star}\right)=\sum_{\ell \in \curlywedge\left(W_{0}+V_{0}\right)} \varphi(x-\ell) \cdot p\left(\ell^{\star}\right) .
$$

For given $x \in G$, the above sum consists of at most one nonzero term. By definition $g$ is uniformly continuous, and we have $g(\ell)=p\left(\ell^{\star}\right)$ for all $\ell \in \curlywedge(W)$. Also note that $g(x)=\mathbf{0}$ if $x \notin \curlywedge\left(W_{0}\right)+U_{0}$ as $p(y)=\mathbf{0}$ for all $y \notin W_{0}$. We show that $g$ is almost periodic by showing that its almost periods are relatively dense. Fix arbitrary $\varepsilon>0$ and choose any symmetric zero neighborhood $V \subset H$ such that $V \subset V_{0}$ and $\left\|p(y)-p\left(y^{\prime}\right)\right\|<\varepsilon$ whenever $y-y^{\prime} \in V$, which is possible due to uniform continuity of $p$. Here $\|\cdot\|$ is any norm on $\mathbb{R}^{d}$. We show that $\curlywedge(V)$ is a set of $\varepsilon$-almost periods for $g$, which proves the claim.

Fix any $t \in \mathcal{\curlywedge}(V)$ and assume first $x \in \mathcal{\curlywedge}\left(W_{0}\right)+U_{0}$. Then, $x=\ell+u$ for some $\ell \in \curlywedge\left(W_{0}\right)$ and some $u \in U_{0}$. Moreover we have $x-t=\ell-t+u$ and $\ell-t \in \mathcal{\lambda}\left(W_{0}+V_{0}\right)$. Hence, we can estimate

$\|g(x-t)-g(x)\|=\left\|\varphi(u) \cdot p\left(\ell^{\star}-t^{\star}\right)-\varphi(u) \cdot p\left(\ell^{\star}\right)\right\| \leq\left\|p\left(\ell^{\star}-t^{\star}\right)-p\left(\ell^{\star}\right)\right\|<\varepsilon$.

Similarly, assume $x-t \in \curlywedge\left(W_{0}\right)+U_{0}$. Then, $x-t=\ell+u$ for some $\ell \in \curlywedge\left(W_{0}\right)$ and some $u \in U_{0}$. Moreover we have $x=\ell+t+u$ and $\ell+t \in \mathcal{\curlywedge}\left(W_{0}+V_{0}\right)$. Hence, we can estimate

$$
\|g(x-t)-g(x)\| \leq\left\|p\left(\ell^{\star}\right)-p\left(\ell^{\star}+t^{\star}\right)\right\|<\varepsilon .
$$

We are left with the case $x \notin \curlywedge\left(W_{0}\right)+U_{0}$ and $x-t \notin \curlywedge\left(W_{0}\right)+U_{0}$. But then $\|g(x-t)-g(x)\|=\|\mathbf{0}-\mathbf{0}\|=0$. 
The function $w$ can be constructed analogously. In fact, for $w$ the above construction works on general LCAG.

Let us summarize the above two results in the following theorem.

Theorem 5 Every modulation of a weighted model set is a deformed weighted model set in the extended CPS from Lemma 4. In Euclidean space, the class of deformed weighted model sets coincides with the class of modulated weighted model sets.

\section{Ideal crystals and their modulations}

Ideal crystals are mathematical models of physical crystals. Given a physical crystal, the fundamental problem of crystallography is reconstructing its period group and a decoration of the fundamental domain from diffraction data.

In this section we will show that ideal crystals and their modulations have a strongly almost periodic Dirac comb. Ideal crystals are model sets having a discrete internal space. We also show that the class of ideal crystals is stable under so-called commensurate modulation.

Definition 5 A locally finite set is an ideal crystal if its period group is a lattice.

The period group $P(\Lambda)$ of $\Lambda \subset G$ is given by $P(\Lambda)=\{t \in G: t+\Lambda=\Lambda\}$. The above definition is in line with [37, Def. 5.1] and [42, Def. 1.3], see also the following lemma. An ideal crystal is called a crystallographic point set in [3, Def. 3.1].

\subsection{Parametrization}

The following characterization of ideal crystal is certainly well-known, see, e.g., [3, Prop. 3.1]. We provide the short proof for the reader's convenience, as the argument shows how to construct a torus parametrization for the hull of an ideal crystal.

Lemma 5 Let $\Lambda \subset G$ be locally finite. Then, $\Lambda$ is an ideal crystal if and only if there exist a lattice $\Gamma$ and a finite set $F$ such that $\Lambda=\Gamma+F$.

Proof " $\Rightarrow$ ": Assume that the period group $P$ of $\Lambda$ is a lattice. Let $D$ be any relatively compact fundamental domain of $P$. Choosing representatives within $D$, we get

$$
\Lambda=\bigcup_{\lambda \in \Lambda}(P+\lambda)=\bigcup_{\lambda \in \Lambda \cap D}(P+\lambda)=P+(\Lambda \cap D)
$$

As $\Lambda \cap D$ is a finite set due to local finiteness of $\Lambda$, this shows $\Lambda=\Gamma+F$ with $\Gamma=P$ and $F=\Lambda \cap D$.

" $\Leftarrow$ ": Note that the period group $P$ of $\Lambda=\Gamma+F$ satisfies $\Gamma \subset P \subset \Gamma+F-F$. Hence, the group $P$ is both discrete and relatively dense. This means that $P$ is a lattice. In particular $\Lambda$ is an ideal crystal. 
Remark 7 The decomposition $\Lambda=\Gamma+F$ may not be unique. A simple example is $\Lambda=\mathbb{Z}+\{0,1 / 2\}=1 / 2 \cdot \mathbb{Z}$, which satisfies $\Lambda=P(\Lambda)$. In general, we have $\Gamma \subset P(\Lambda)$, and the above proof shows that we may choose $\Gamma=P(\Lambda)$ and $F=\Lambda \cap D$, where $D$ is a relatively compact fundamental domain of $P(\Lambda)$ in $G$. In that case, $\Lambda$ is uniquely parametrized by $\Gamma$ and $F$, i.e., we have the direct sum decomposition $\Lambda=$ $\Gamma \oplus F$, compare [3, Prop. 3.1]. That decomposition is canonical from an experimental viewpoint.

It follows from the previous remark that we can parametrize the hull of $\Lambda$ by the compact group $G / \Lambda(G)$. Indeed, $x+P(\Lambda) \mapsto x+\Lambda$ provides a homeomorphism which commutes with the natural shift action. Thus $\delta_{\Lambda}$ is strongly almost periodic by Lemma 2, and the Bragg peaks in the diffraction spectrum are indexed by the dual of $P(\Lambda)$.

Strong almost periodicity of ideal crystals was already proved in [37, Cor. 5.6] and in [2] by a different method. The latter reference also gives a partial converse. Recall that $\Lambda \subset G$ has finite local complexity if $\Lambda-\Lambda$ is uniformly discrete.

Proposition 6 [37, Cor. 5.6] Let $\Lambda$ be a Delone set in a compactly generated LCAG $G$. If $\Lambda$ has finite local complexity and $\delta_{\Lambda}$ is strongly almost periodic, then $\Lambda$ is an ideal crystal.

\subsection{Ideal crystals and model sets}

Ideal crystals can be characterized as model sets having discrete internal space. The parametrization as a model set can be used to compute the diffraction of an ideal crystal by the diffraction formula for model sets.

Proposition 7 (Ideal crystals and model sets)

(i) Any model set from a CPS with discrete internal space is an ideal crystal.

(ii) Any ideal crystal is a model set in some CPS with discrete internal space.

Proof "(i)" Consider any CPS $(G, H, \mathcal{L})$ with discrete $H$ and relatively compact nonempty window $W \subset H$. The group $\Gamma=\curlywedge(\{0\})$ is a model set as $H$ is discrete. Thus $\Gamma$ is a Delone set and hence, a lattice in $G$. Moreover, for all $t \in \Gamma$ we have

$$
t+\curlywedge(W)=\curlywedge\left(t^{\star}+W\right)=\curlywedge(0+W)=\curlywedge(W) .
$$

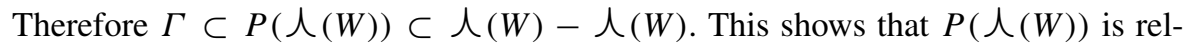
atively dense and uniformly discrete, thus a lattice. As $\curlywedge(W)$ is locally finite, this shows that $\curlywedge(W)$ is an ideal crystal.

"(ii)" Assume that $\Lambda=\Gamma+F$ for some lattice $\Gamma$ and some non-empty finite set $F$. Consider the group $H=G / \Gamma$ equipped with the discrete topology and let $\mathcal{L}=\{(x, \bar{x}): x \in G\}$, where $\bar{x}=x+\Gamma$. We claim that $(G, H, \mathcal{L})$ is a CPS.

Let us first prove that the group $\mathcal{L}$ is a lattice. The group $\mathcal{L}$ is discrete: Since $\Gamma$ is a lattice in $G$, we can find an open zero neighborhood $U \subset G$ such that $\Gamma \cap U=$ \{0\}. Since $\{\overline{0}\}$ is open in $H$ by discreteness of $H$, the set $U \times\{\overline{0}\}$ is an open zero 
neighborhood in $G \times H$. Now consider any point $(y, \bar{y}) \in \mathcal{L} \cap(U \times\{\overline{0}\})$. Then, $\bar{y}=\overline{0}$, which implies $y \in \Gamma$. Thus $y \in \Gamma \cap U$, which implies $y=0$. Thus $\mathcal{L}$ is discrete. The group $\mathcal{L}$ is relatively dense: Let $K \subset G$ be compact such that $\Gamma+K=G$. Let $(x, \bar{y}) \in G \times H$ be arbitrary. We can write $x=z_{1}+k_{1}, y=z_{2}+k_{2}$ for some $z_{i} \in \Gamma$ and $k_{i} \in K$. Note that $\bar{x}=\overline{k_{1}}$ and $\bar{y}=\overline{k_{2}}$. Then,

$$
(x, \bar{y})=\left(z_{1}+k_{1}, \overline{k_{2}}\right)=\left(z_{1}+k_{2}, \overline{z_{1}+k_{2}}\right)+\left(k_{1}-k_{2}, \overline{0}\right) \in \mathcal{L}+((K-K) \times\{\overline{0}\}) .
$$

This shows that $\mathcal{L}$ is relatively dense. We have shown that $\mathcal{L}$ is a lattice.

It is obvious from the definition of $\mathcal{L}$ that $\left.\pi^{G}\right|_{\mathcal{L}}$ is one-to-one, and that $\pi^{H}(\mathcal{L})=H$ is dense in $H$. Hence, $(G, H, \mathcal{L})$ is a CPS.

Now let $W=\bar{F}$. Then, $W$ is compact as $F$ is finite. Furthermore, we have $\curlywedge(W)=$ $\{x \in G: \bar{x} \in \bar{F}\}=F+\Gamma$.

Consider an ideal crystal $\Lambda=\Gamma+F=\curlywedge(W)$ as in the proof of (ii). Then, $P(\Lambda)^{\star}=P_{W}$, with $P_{W}=\{h \in H: h+W=W\}$ the period group of the window. As a simple consequence $P(\Lambda)=\Gamma$ if and only if the window is aperiodic, i.e., $P_{W}=\{\overline{0}\}$. Hence, any ideal crystal can be realized as a model set with aperiodic window. In that case, the torus parametrization of Theorem 3 is an isomorphism by [45, Prop. 13.2], which is in line with the results from Sect. 9.1. Injectivity properties of the torus parametrization in the case of nonzero window periods are discussed in [39].

\subsection{Modulations and deformations of ideal crystals}

As any ideal crystal is a model set, we can consider weighted and deformed variants of it. As the internal space can be chosen to be discrete, the following result is readily inferred from Sect. 8.

Theorem 6 Let $\Lambda$ be an ideal crystal, described as a model set in the extended CPS $(G, H, \mathcal{L})$ from Lemma 4 . Then, any modulation of $\Lambda$ is a deformed weighted variant of the model set $\Lambda$ in $(G, H, \mathcal{L})$. In particular, any modulation of $\Lambda$ is strongly almost periodic. In Euclidean space, the class of modulations of $\Lambda$ coincides with the class of deformed weighted variants of the model set $\Lambda$ in $(G, H, \mathcal{L})$. The classes of deformed weighted model sets in $(G, H, \mathcal{L})$ and of modulations of $\Lambda$ are both modulation stable.

The class of ideal crystals is stable under a restricted class of modulations, which is sometimes called commensurate modulations. A function on $G$ is called fully periodic or crystallographic, if its period group is a lattice in $G$.

Proposition 8 Let $\Lambda=\Gamma+F$ be an ideal crystal, and let $g: G \rightarrow G$ be any function such that $\left.g\right|_{\Gamma}$ is fully periodic. Then, $\Lambda^{g}=\{\lambda+g(\lambda): \lambda \in \Lambda\}$ is an ideal crystal.

Proof Given $\Lambda=\Gamma+F$, let $L=\left\{\gamma \in \Gamma: T_{\gamma} g=g\right\}$ be the period group of $g$ in $\Gamma$. As $\left.g\right|_{\Gamma}$ is fully periodic, $L \subset \Gamma$ is a lattice. In particular, $L$ has finite index in $\Gamma$. To 
see the latter statement, write $L+K=G$ for some compact set $K$. We then have

$$
\Gamma=\Gamma \cap(L+K) \subset L+K \cap \Gamma \subset \Gamma .
$$

For the first inclusion, assume $\ell+k \in \Gamma$ for $\ell \in L \subset \Gamma$ and $k \in K$. Then, $k \in \Gamma-\ell \subset \Gamma-L \subset \Gamma-\Gamma=\Gamma$, and the inclusion follows. As $K \cap \Gamma$ is a finite set, this shows that $L$ has finite index in $\Gamma$. We can thus write $\Gamma=L+E$ for a lattice $L$ and a finite set $E$. Now define the finite set

$$
F_{g}=\{e+f+g(e+f): e \in E, f \in F\} .
$$

Then, we have

$$
\begin{aligned}
\Lambda^{g} & =\{\ell+e+f+g(\ell+e+f): \ell \in L, e \in E, f \in F\} \\
& =\{\ell+e+f+g(e+f): \ell \in L, e \in E, f \in F\} \\
& =L+F_{g} .
\end{aligned}
$$

This shows that $\Lambda^{g}$ is an ideal crystal.

Remark 8 If $\left.g\right|_{\Gamma}$ is fully periodic, then $g(\Gamma)$ is finite. The converse is true if $\left.g\right|_{\Gamma}$ is almost periodic since for $g(\Gamma)$ finite, any $\varepsilon$-almost period is a period for sufficiently small $\varepsilon$ in that case. Sometimes, the case $g(\Gamma)$ finite is referred to as commensurate modulation.

Remark 9 By Remark 7, we may canonically write $\Lambda^{g}=P\left(\Lambda^{g}\right) \oplus\left(D \cap \Lambda^{g}\right)$, where $D$ is a relatively compact fundamental domain of the lattice $P\left(\Lambda^{g}\right)$. The lattice $L$ used in the above proof might not coincide with $P\left(\Lambda^{g}\right)$.

Remark 10 The previous result shows that any commensurate lattice modulation with trivial weight function is an ideal crystal. In this sense, ideal crystals naturally arise from lattices by commensurate modulation. Conversely, in $G=\mathbb{R}^{d}$ it is not too difficult to see that any ideal crystal is a commensurate lattice modulation.

Acknowledgements J.-Y. Lee would like to thank to Hyeong-Chai Jeong for motivating this work. J.-Y. Lee was supported by NRF Grant No. 2019R1I1A3A01060365. N. Strungaru was supported by NSERC with Grants 03762-2014 and 2020-00038, and he is grateful for the support. We thank the referees for very detailed suggestions for improvement.

Funding Open Access funding enabled and organized by Projekt DEAL.

Open Access This article is licensed under a Creative Commons Attribution 4.0 International License, which permits use, sharing, adaptation, distribution and reproduction in any medium or format, as long as you give appropriate credit to the original author(s) and the source, provide a link to the Creative Commons licence, and indicate if changes were made. The images or other third party material in this article are included in the article's Creative Commons licence, unless indicated otherwise in a credit line to the material. If material is not included in the article's Creative Commons licence and your intended use is not permitted by statutory regulation or exceeds the permitted use, you will need to obtain permission directly from the copyright holder. To view a copy of this licence, visit http://creativecommons.org/licenses/by/4.0/. 


\section{A Group valued almost periodic functions}

We discuss almost periodicity for functions which take values in a topological abelian group. This subsumes real or complex-valued almost periodic functions, see [14,18,35] for standard treatments. It also subsumes almost periodic functions with values in Euclidean space [71], see also [28, Sec. 33.26-27]. ${ }^{5}$ Our results are natural extensions from the case of complex-valued functions. In view of our applications, we restrict analysis to uniformly continuous and bounded functions.

\section{A.1 Function space topology}

The topology on our function spaces is induced by the uniform structure of uniform convergence. For background about topologies on function spaces, see, e.g., Chapters 6 and 7 in [36] or Chapters II, III.3 and X.1 in [16].

Let $G, H$ be LCAG, where $H$ is complete. Consider the set $\mathcal{H}$ of all maps from $G$ to $H$. For each zero neighborhood $W \subset H$ define

$$
U_{W}=\left\{\left(h_{1}, h_{2}\right) \in \mathcal{H} \times \mathcal{H}: h_{1}(x)-h_{2}(x) \in W \text { for all } x \in G\right\}
$$

and consider $\mathcal{U}=\left\{U_{W}: W\right.$ is a zero neighborhood in $\left.H\right\}$. The following facts are standard.

Lemma 6 (i) $\mathcal{U}$ is a fundamental set of entourages for a uniformity on $\mathcal{H}$.

(ii) $\mathcal{H}$ is complete with respect to the above uniformity.

(iii) The subspaces $C(G, H)$ of continuous functions, $C_{b}(G, H)$ of continuous and bounded functions and $C_{u}(G, H)$ of uniformly continuous and bounded functions are closed in $\mathcal{H}$.

Note that for $H=\mathbb{C}$ with its usual topology, the topology on the function space $\mathcal{H}$ induced by $\mathcal{U}$ coincides with the topology induced by the supremum norm.

\section{A.2 Almost periodic functions}

From now on, we restrict to uniformly continuous and bounded functions. In that setting, almost periodicity can be described in three equivalent ways.

For $f \in C_{\mathrm{u}}(G, H)$ consider its hull $G_{f}$, i.e., the closure of the translation orbit of $f$ in the topology of uniform convergence. We write

$$
G_{f}=\overline{\left\{T_{t} f: t \in G\right\}} \subset C_{\mathrm{u}}(G, H),
$$

where we use the notation $\left(T_{t} f\right)(x)=f(-t+x)$ for function translation. We say that $f$ is Bochner almost periodic, if its hull $G_{f}$ is compact.

\footnotetext{
5 In that context, let us mention methods of sampling along almost periodic sequences [5], which complement dynamical systems techniques and may also be put into a group setting.
} 
We say $f$ is Bohr almost periodic, if for every zero neighborhood $W \subset H$ the set

$$
P_{W}(f)=\left\{t \in G:\left(T_{t} f, f\right) \in U_{W}\right\}
$$

of $W$-almost periods of $f$ is relatively dense. Here $A \subset G$ is relatively dense in $G$ if there is a compact set $K \subset G$ such that $A+K=G$.

Almost periodicity can also be defined using the Bohr compactification $G_{\mathrm{b}}$ of $G$. This is the dual group of the dual group $(\widehat{G})_{d}$ of $G$, equipped with the discrete topology. See [24, Sec. 4.7] for background. We denote by $i_{\mathrm{b}}: G \rightarrow G_{\mathrm{b}}$ the canonical injection map.

Proposition 9 Consider $f \in C_{u}(G, H)$. Then, the following are equivalent.

(i) $f$ is Bochner almost periodic.

(ii) $f$ is Bohr almost periodic.

(iii) There exists $f_{b} \in C\left(G_{b}, H\right)$ such that $f=f_{b} \circ i_{b}$.

A proof can be given by properly adapting the arguments from the case $H=\mathbb{C}$, which are given, e.g., in [52]. As arguments based on the Bohr compactification appear throughout the article, we write out the proof $(i) \Leftrightarrow$ (iii) for the reader's convenience, assuming that we have established $(i) \Leftrightarrow(i i)$.

Proof $(i) \Rightarrow($ iii $)$ : Consider the map $T f: G \rightarrow G_{f}$ given by $t \mapsto(T f)(t)=T_{t} f$. It is a continuous group homomorphism. As $G_{f}$ is a compact by assumption, we can use the universal property of the Bohr compactification to infer the existence of a continuous group homomorphism $(T f)_{\mathrm{b}}: G_{\mathrm{b}} \rightarrow G_{f}$ satisfying $T f=(T f)_{\mathrm{b}} \circ i_{\mathrm{b}}$. Let us consider the evaluation map $\delta: G_{f} \rightarrow H$, which is defined by $\delta(g)=g(0)$ for $g \in G_{f}$. We then have $f=\delta \circ T f$. The situation is summarized in the following commutative diagram.

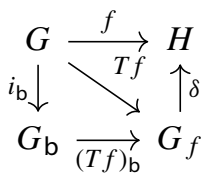

Now define $f_{\mathrm{b}}: G_{\mathrm{b}} \rightarrow H$ by $f_{\mathrm{b}}=\delta \circ(T f)_{\mathrm{b}}$. Then, $f=f_{\mathrm{b}} \circ i_{\mathrm{b}}$.

(iii) $\Rightarrow($ i $)$ : Consider $f_{\mathrm{b}} \in C\left(G_{\mathrm{b}}, H\right)$ such that $f=f_{\mathrm{b}} \circ i_{\mathrm{b}}$. Consider any zero neighborhood $W \subset H$ and choose some zero neighborhood $V \subset H$ such that $\bar{V} \subset W$. As $G_{\mathrm{b}}$ is a compact group, the function $f_{\mathrm{b}} \in C\left(G_{\mathrm{b}}, H\right)$ is Bohr almost periodic and hence also Bochner almost periodic by $(i i) \Rightarrow(i)$. For some finite set $F_{\mathrm{b}} \subset G_{\mathrm{b}}$ we thus have

$$
\left\{T_{t_{\mathrm{b}}} f_{\mathrm{b}}: t_{\mathrm{b}} \in G_{\mathrm{b}}\right\} \subset \bigcup_{t_{\mathrm{b}} \in F_{\mathrm{b}}} U_{V}\left(T_{t_{\mathrm{b}}} f_{\mathrm{b}}\right)
$$


Now observe $U_{V}\left(T_{t_{\mathrm{b}}} f_{\mathrm{b}}\right) \circ i_{\mathrm{b}} \subset U_{V}\left(T_{t_{\mathrm{b}}} f_{\mathrm{b}} \circ i_{\mathrm{b}}\right)$ and $T_{t} f=\left(T_{i_{\mathrm{b}}(t)} f_{\mathrm{b}}\right) \circ i_{\mathrm{b}}$. Hence, the above implies

$$
\begin{aligned}
\left\{T_{t} f: t \in G\right\} & =\left\{T_{i_{\mathrm{b}}(t)} f_{\mathrm{b}}: t \in G\right\} \circ i_{\mathrm{b}} \subset \bigcup_{t_{\mathrm{b}} \in F_{\mathrm{b}}} U_{V}\left(T_{t_{\mathrm{b}}} f_{\mathrm{b}}\right) \circ i_{\mathrm{b}} \\
& \subset \bigcup_{t \in F} U_{W}\left(T_{i_{\mathrm{b}}(t)} f_{\mathrm{b}}\right) \circ i_{\mathrm{b}} \subset \bigcup_{t \in F} U_{W}\left(T_{t} f\right),
\end{aligned}
$$

where $F \subset G$ is some finite set. For the second inclusion we used that $i_{\mathrm{b}}(G) \subset G_{\mathrm{b}}$ is dense in $G_{\mathrm{b}}$. As $W \subset H$ was an arbitrary zero neighborhood, this shows total boundedness of the hull. As $C_{\mathrm{u}}(G, H)$ is complete, this implies compactness of the hull [36, Ch. 6.32].

\section{A.3 Almost periodic functions on subgroups}

Let $L$ be a closed subgroup of an LCAG $G$, and let $H$ be an LCAG. We discuss the relation between almost periodic functions $G \rightarrow H$ and $L \rightarrow H$. It is advantageous to perform the analysis on the Bohr compactifications $L_{\mathrm{b}}$ and $G_{\mathrm{b}}$, since we can then work with continuous functions on compact groups. The natural continuous injection maps lead to the following commutative diagram.

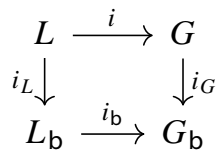

Lemma 7 The above continuous group homomorphism $i_{b}$ is uniquely defined. It is an embedding of $L_{b}$ into $G_{b}$.

Proof Consider the following commutative diagram.

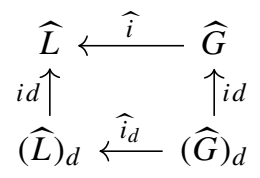

Here $\widehat{i}: \widehat{G} \rightarrow \widehat{L}$ denotes the character restriction map, and $(\widehat{L})_{d}$ resp. $(\widehat{G})_{d}$ denote the groups $\widehat{L}$ resp. $\widehat{G}$ equipped with the discrete topology. As $L_{\mathrm{b}}=\widehat{(\widehat{L})_{d}}$ and $G_{\mathrm{b}}=\widehat{(\widehat{G})_{d}}$, dualizing this diagram yields the first part of the claim. We show that $i_{\mathrm{b}}=\widehat{i_{d}}$ is oneto-one: Note first that the character restriction map is onto [55, Thm. 4.2.14], which follows from Pontryagin duality $\widehat{L} \simeq \widehat{G} / L_{0}$, where $L_{0} \subset \widehat{G}$ is the annihilator of $L$, compare [55, Thm. 4.2.21]. Then, $\widehat{i}_{d}:(\widehat{G})_{d} \rightarrow(\widehat{L})_{d}$ is a continuous onto group homomorphism. In particular, $\widehat{i}_{d}$ has dense range. Now we get by Pontryagin duality that $i_{\mathrm{b}}=\widehat{i_{d}}$ is one-to-one.

Using Proposition 9, we can derive the following two results. 
Lemma 8 Let $f \in C_{u}(G, H)$ be an almost periodic function and let $L$ be a closed subgroup of $G$. Consider the restriction $f_{L}$ of $f$ to $L$. Then, $f_{L} \in C_{u}(L, H)$ is almost periodic on $L$.

Proof The almost periodic $f \in C_{\mathrm{u}}(G, H)$ can be written as $f=F \circ i_{G}$ for a unique continuous $F: G_{\mathrm{b}} \rightarrow H$. Then, $F_{L}: L_{\mathrm{b}} \rightarrow H$, defined by $F_{L}=F \circ i_{\mathrm{b}}$, is continuous. Then, $F_{L} \circ i_{L}: L \rightarrow H$ is almost periodic on $L$ and satisfies $F_{L} \circ i_{L}(l)=$ $F \circ i_{\mathrm{b}} \circ i_{L}(l)=F \circ i_{G} \circ i(l)=f(l)$. We have shown $F_{L} \circ i_{L}=f_{L}$, with $f_{L}$ the restriction of $f$ to $L$.

Lemma 9 Let L be a closed subgroup of $G$. Consider $H=\mathbb{R}^{d}$ and let $f_{L} \in C_{u}(L, H)$ be an almost periodic function. Then, there exists an almost periodic extension $f \in$ $C_{u}(G, H)$ of $f_{L}$ to $G$.

Remark 11 The above extension might not be unique.

Proof The almost periodic function $f_{L} \in C_{\mathrm{u}}(L, H)$ can be written as $f_{L}=F_{L} \circ i_{L}$ for a unique continuous function $F_{L}: L_{\mathrm{b}} \rightarrow H$. Consider $A=i_{\mathrm{b}}\left(L_{\mathrm{b}}\right) \subset G_{\mathrm{b}}$. We can define a continuous function $F_{A}: A \rightarrow H$ by $F_{A}(g)=F_{L}(l)$, where $l \in L_{\mathrm{b}}$ is uniquely determined by $i_{\mathrm{b}}(l)=g$, as $i_{\mathrm{b}}: L_{\mathrm{b}} \rightarrow A$ is a homeomorphism, compare Lemma 7. As $A$ is closed, we can invoke Tietze's extension theorem [22, Thm. A.8.3] on each component of $F_{A}$ to infer that $F_{A}$ admits a continuous extension $F: G_{\mathrm{b}} \rightarrow H$. Hence, $f \in C_{\mathrm{u}}(G, H)$ defined by $f=F \circ i_{G}$ is an almost periodic extension of $f_{L}$ to $G$.

\section{References}

1. Argabright, L.N., Gil de Lamadrid, J.: Fourier Analysis of Unbounded Measures on Locally Compact Abelian Groups. Mem. Am. Math. Soc. 145. American Mathematical Society, Providence, RI (1974)

2. Aujogue, J.-B., Barge, M., Kellendonk, J., Lenz, D.: Equicontinuous factors, proximality and Ellis semigroup for Delone sets. In: Kellendonk, J., Lenz, D., Savinien, J. (eds.) Mathematics of Aperiodic Order. Progr. Mathe. 309. Birkhäuser/Springer, Basel, pp. 137-194 (2015)

3. Baake, M., Grimm, U.: Aperiodic Order, Volume 1: A Mathematical Invitation, Encyclopedia of Mathematics and Its Applications, vol. 149. Cambridge University Press, Cambridge (2013)

4. Baake, M., Grimm, U. (eds.): Aperiodic Order, Volume 2: Crystallography and Almost Periodicity, Encyclopedia of Mathematics and Its Applications, vol. 166. Cambridge University Press, Cambridge (2017)

5. Baake, M., Haynes, A., Lenz, D.: Averaging almost periodic functions along exponential sequences, in: [4], pp. 343-362 (2017)

6. Baake, M., Hermisson, J., Pleasants, P.A.B.: The torus parametrization of quasiperiodic LI classes. J. Phys. A 30, 3029-3056 (1997)

7. Baake, M., Lenz, D.: Dynamical systems on translation bounded measures: pure point dynamical and diffraction spectra. Ergod. Theory Dyn. Syst. 24, 1867-1893 (2004)

8. Baake, M., Lenz, D.: Deformation of Delone dynamical systems and pure point diffraction. J. Fourier Anal. Appl. 11, 125-150 (2005)

9. Baake, M., Lenz, D., Moody, R.V.: Characterization of model sets by dynamical systems. Ergod. Theory Dyn. Syst. 27, 341-382 (2007)

10. Baake, M., Moody, R.V. (eds.): Directions in Mathematical Quasicrystals. CRM Monogr. Ser. 13. American Mathematical Society, Providence, RI (2000)

11. Baake, M., Moody, R.V.: Weighted Dirac combs with pure point diffraction. J. Reine Angew. Math. (Crelle) 573, 61-94 (2004) 
12. Berg, C., Forst, G.: Potential Theory on Locally Compact Abelian Groups. Springer, Berlin (1975)

13. Bernuau, G., Duneau, M.: Fourier analysis of deformed model sets, in: [10], pp. 43-60 (2000)

14. Besicovitch, A.S.: Almost Periodic Functions. Dover Publications, New York (1955)

15. Bombieri, E., Taylor, J.E.: Quasicrystals, tilings, and algebraic number theory: some preliminary connections. In: L. Keen (ed.) The Legacy of Sonya Kovalevskaya. Contemp. Math. 64. American Mathematical Society, Providence, RI, pp. 241-264 (1987)

16. Bourbaki, N.: General Topology. Springer, Berlin (1998)

17. Brouns, E., Visser, J.W., de Wolff, P.M.: An anomaly in the crystal structure of $\mathrm{Na}_{2} \mathrm{CO}_{3}$. Acta Crystallogr. 17, 614 (1964)

18. Corduneanu, C.: Almost Periodic Functions, Interscience Tracts in Pure and Applied Mathematics, vol. 22. Interscience Publishers, New York (1968)

19. Cowley, J.M.: Diffraction Physics, 3rd edn. North-Holland, Amsterdam (1995)

20. de Bruijn, N.G.: Modulated quasicrystals. Nederl. Akad. Wetensch. Indag. Math. 49, 121-132 (1987)

21. de Wolff, P.M.: The pseudo-symmetry of modulated crystal structures. Acta Crystallogr. A30, 777-785 (1974)

22. Deitmar, A., Echterhoff, S.: Principles of Harmonic Analysis. Springer, New York (2009)

23. Duneau, M., Katz, A.: Quasiperiodic patterns. Phys. Rev. Lett. 54, 2688-1691 (1985)

24. Folland, G.: A Course in Abstract Harmonic Analysis. CRC Press, Boca Raton (1995)

25. Frettlöh, D., Richard, C.: Dynamical properties of almost repetitive Delone sets. Discrete Contin. Dyn. Syst. 34, 533-558 (2014)

26. Gil de Lamadrid, J., Argabright, L.N.: Almost Periodic Measures. Mem. Am. Math. Soc. 428. American Mathematical Society, Providence, RI (1990)

27. Gouéré, J.-B.: Quasicrystals and almost periodicity. Commun. Math. Phys. 255, 655-681 (2005)

28. Hewitt, E., Ross, K.A.: Abstract Harmonic Analysis, Vol. II, Grundlehren der Mathematischen Wissenschaften [Fundamental Principles of Mathematical Sciences] 152. Springer, Berlin (1970)

29. Hof, A.: On diffraction by aperiodic structures. Commun. Math. Phys. 169, 25-43 (1995)

30. Hof, A.: Diffraction by aperiodic structures, in: [49], pp. 239-268 (1997)

31. Janner, A., Janssen, T.: Symmetry of periodically distorted crystals. Phys. Rev. B 15, 643-658 (1977)

32. Janner, A., Janssen, T.: Aperiodic crystals and superspace concepts. Acta Crystallogr. B70, 617-651 (2014)

33. Janssen, T., Chapuis, G., de Boissieu, M.: Aperiodic Crystals. From Modulated Phases to Quasicrystals. IUCr Monographs on Crystallography 20. Oxford University Press (2007)

34. Janssen, T.: A brief history of aperiodic crystals: 1962-2012. In: Schmid, S., Withers, R.L., Lifshitz, R. (eds.) Aperiodic Crystals, pp. 1-9. Springer, Dordrecht (2013)

35. Katznelson, Y.: An Introduction to Harmonic Analysis, 3rd edn. Cambridge Mathematical Library, Cambridge University Press, Cambridge (2004)

36. Kelley, J.L.: General Topology. von Nostrand, Princeton (1955)

37. Kellendonk, J., Lenz, D.: Equicontinuous Delone dynamical systems. Can. J. Math. 65, 149-170 (2013)

38. Keller, G., Richard, C.: Dynamics on the graph of the torus parametrisation. Ergod. Theory Dyn. Syst. 28, 1048-1085 (2018)

39. Keller, G., Richard, C.: Periods and factors of weak model sets. Isr. J. Math. 229, 85-132 (2019)

40. Kramer, P., Neri, R.: On periodic and non-periodic space fillings of $\mathbb{E}^{m}$ obtained by projection. Acta Crystallogr. A 40, 580-587 (1984), and Erratum, Acta Crystallogr. A 41, 619 (1985)

41. Kuipers, L., Niederreiter, H.: Uniform Distribution of Sequences. Pure and Applied Mathematics. Wiley, New York (1974)

42. Lagarias, J.C.: Mathematical quasicrystals and the problem of diffraction, in: [10], pp. 61-93 (2000)

43. Lovelace, J.J., et al.: Protein crystals can be incommensurately modulated. J. Appl. Crystallogr. 41, 600-605 (2008)

44. Lenz, D.: Continuity of eigenfunctions of uniquely ergodic dynamical systems and intensity of Bragg peaks. Commun. Math. Phys. 287, 225-258 (2009)

45. Lenz, D., Richard, C.: Pure point diffraction and cut and project schemes for measures: the smooth case. Math. Z. 256, 347-378 (2007)

46. Lenz, D., Strungaru, N.: Pure point spectrum for measure dynamical systems on locally compact abelian groups. J. Math. Pures Appl. 92, 323-341 (2009)

47. Lenz, D., Strungaru, N.: On weakly almost periodic measures. Trans. Am. Math. Soc. 371, 6843-6881 (2019)

48. Meyer, Y.: Algebraic Numbers and Harmonic Analysis. North-Holland, Amsterdam (1972) 
49. Moody, R.V. (ed.): The Mathematics of Long-Range Aperiodic Order, NATO ASI Series C 489. Kluwer, Dordrecht (1997)

50. Moody, R.V.: Meyer sets and their duals, in: [49], pp. 403-441 (1997)

51. Moody, R.V.: Model sets: a survey. In: Axel, F., Dénoyer, F., Gazeau, J.-P. (eds.) From Quasicrystals to More Complex Systems. Centre de Physique des Houches 13. Springer, Berlin, pp. 145-166 (2000)

52. Moody, R.V., Strungaru, N.: Almost Periodic Measures and their Fourier Transforms, in: [4], pp. 173-270 (2017)

53. Pedersen, G.K.: Analysis Now, Graduate Texts in Mathematics 118. Springer, New York (1989); rev. printing (1995)

54. Reich, A.: Präkompakte Gruppen und Fastperiodizität. Math. Z. 116, 218-234 (1970)

55. Reiter, H., Stegeman, J.D.: Classical Harmonic Analysis and Locally Compact Groups. Clarendon Press, Oxford (2000)

56. Richard, C., Strungaru, N.: A short guide to pure point diffraction in cut-and-project sets. J. Phys. A 50, 154003 (2017)

57. Richard, C., Strungaru, N.: Pure point diffraction and Poisson summation. Ann. Henri Poincaré 18, 3903-3931 (2017)

58. Richard, C.: Dense Dirac combs in Euclidean space with pure point diffraction. J. Math. Phys. 44, 4436-444 (2003)

59. Robinson Jr., E.A.: The dynamical properties of Penrose tilings. Trans. Am. Math. Soc. 348, 4447-4464 (1996)

60. Schlottmann, M.: Generalized model sets and dynamical systems, in: [10], pp. 143-159 (2000)

61. Simon, B.: Representations of Finite and Compact Groups, Graduate Studies in Mathematics, vol. 10. American Mathematical Society, Providence, RI (1996)

62. Sing, B., Welberry, T.R.: Deformed model sets and distorted Penrose tilings. Z. Krist. 221, 621-634 (2006)

63. Sing, B.: Modulated quasicrystals. Z. Krist. 223, 765-769 (2008)

64. Souvignier, B.: Ted Janssen (1936-2017). Acta Crystallogr. 74, 403-404 (2018)

65. Strungaru, N.: Almost periodic pure point measures, in: [4], pp. 271-342 (2017)

66. Strungaru, N.: On the Fourier analysis of measures with Meyer set support. J. Funct. Anal. 278, 108404 (2020)

67. van Smaalen, S.: Incommensurate Crystallography, IUCr Monographs on Crystallography, vol. 21. Oxford University Press, Oxford (2007)

68. Veech, W.A.: Almost automorphic functions on groups. Am. J. Math. 87, 719-751 (1965)

69. Walters, P.: An Introduction to Ergodic Theory. Springer, New York (1982)

70. Welberry, T.R., Sing, B.: Deformed Penrose tilings. Philos. Mag. 87, 2877-2886 (2007)

71. Zaidman, S.: Almost Periodic Functions in Abstract Spaces, RNM 126. Pitman, Boston (1985)

Publisher's Note Springer Nature remains neutral with regard to jurisdictional claims in published maps and institutional affiliations.

\title{
Affiliations
}

\section{Jeong-Yup Lee ${ }^{1,2} \cdot$ Daniel Lenz $^{3} \cdot$ Christoph Richard $^{4}$ (1) Bernd Sing ${ }^{5}$. Nicolae Strungaru ${ }^{6,7}$}

\author{
Christoph Richard \\ christoph.richard@fau.de \\ Jeong-Yup Lee \\ jylee@cku.ac.kr \\ Daniel Lenz \\ daniel.lenz@uni-jena.de \\ Bernd Sing \\ bernd.sing@cavehill.uwi.edu
}


Nicolae Strungaru

strungarun@macewan.ca

1 Department of Mathematics Education, Catholic Kwandong University, Gangneung, Gangwon 25601, Korea

2 KIAS, 85 Hoegiro, Dongdaemun-gu, Seoul 02455, Korea

3 Fakultät für Mathematik und Informatik, Friedrich Schiller Universität Jena, 07737 Jena, Germany

4 Department für Mathematik, Friedrich-Alexander-Universität Erlangen-Nürnberg, Cauerstrasse 11, 91058 Erlangen, Germany

5 Department of Mathematics, University of the West Indies, Cave Hill, P.O. Box 64, Bridgetown BB11000, Barbados

6 Department of Mathematical Sciences, MacEwan University, 10700 - 104 Avenue, Edmonton, AB T5J 4S2, Canada

7 Institute of Mathematics "Simon Stoilow", Bucharest, Romania 